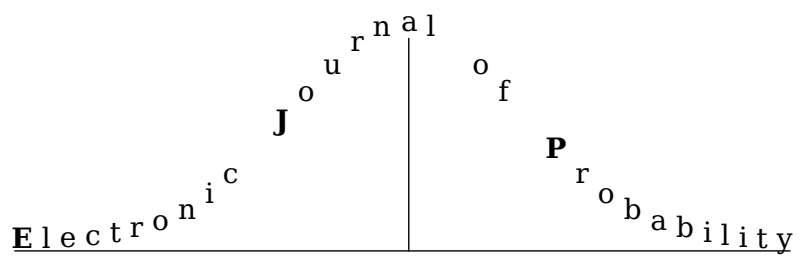

Electron. J. Probab. 25 (2020), no. 17, 1-26.

ISSN: 1083-6489 https://doi.org/10.1214/19-EJP397

\title{
Local bounds for stochastic reaction diffusion equations
}

\author{
Augustin Moinat* Hendrik Weber ${ }^{\dagger}$
}

\begin{abstract}
We prove a priori bounds for solutions of stochastic reaction diffusion equations with super-linear damping in the reaction term. These bounds provide a control on the supremum of solutions on any compact space-time set which only depends on the specific realisation of the noise on a slightly larger set and which holds uniformly over all possible space-time boundary values. This constitutes a space-time version of the so-called "coming down from infinity" property. Bounds of this type are very useful to control the large scale behaviour of solutions effectively and can be used, for example, to construct solutions on the full space even if the driving noise term has no decay at infinity. Our method shows the interplay of the large scale behaviour, dictated by the non-linearity, and the small scale oscillations, dictated by the rough driving noise. As a by-product we show that there is a close relation between the regularity of the driving noise term and the integrability of solutions.
\end{abstract}

Keywords: reaction-diffusion equation; a priori bounds; non-linear stochastic PDE; maximum principle.

AMS MSC 2010: 60H15; 35K57; 35B45.

Submitted to EJP on December 17, 2018, final version accepted on November 15, 2019.

Supersedes arXiv: 1808.10401.

\section{Introduction}

We are interested in reaction diffusion equations of the type

$$
\left(\partial_{t}-\Delta\right) u=-f(u)+\zeta,
$$

over $\mathbb{R}_{t} \times \mathbb{R}_{x}^{d}$ where $\zeta$ is an irregular distribution. The example we have in mind is the case where $\zeta$ is a random noise term, such as space-time white noise for $d=1$, or a noise which is "white in time and coloured in space" for $d \geq 2$. However, we mention right

\footnotetext{
*Imperial College, London, United Kingdom. E-mail: a.moinat@imperial .ac.uk. AM is supported by a PhD grant from University of Warwick.

${ }^{\dagger}$ University of Bath, Bath, United Kingdom. E-mail: h. weber@bath.ac.uk. HW is supported by the Royal Society through the University Research Fellowship UF140187.
} 
away that our main result is purely deterministic and the only information about $\zeta$ that enters is its regularity measured in a suitable space of distributions. The non-linearity $f$ is assumed to be continuous, with super-linear growth at infinity in $u$.

It is well-known that if $f$ satisfies the so-called Osgood condition, that is if $f$ satisfies $\int_{1}^{\infty} \frac{1}{f(u)} d u<\infty$, then solutions of the ODE $\dot{x}=-f(x)$ "come down from infinity in finite time" (see [17]). This means that if $x$ solves the equation over $[0, t]$, then automatically $x(t)$ satisfies a bound which depends on $t$, but holds uniformly over all possible choices of initial datum $x(0)>0$. Similar statements can be derived for reaction diffusion equations based on a comparison principle (see e.g. [19, Chapter 14]) and also stochastic reaction diffusion equations (see e.g. [4, Theorem 6.2.3] and [5]). These bounds are powerful tools to study the long-time behaviour of solutions, both in the deterministic and in the stochastic setting - see e.g. [20] for a construction of invariant measures for stochastic PDEs based on such bounds.

Our main result, Theorem 3.1, is a space-time version of such a bound for solutions of (1.1) with $f(u)=u|u|^{m-1}+g$ where $g$ is bounded. We consider a continuous functions $u: \mathbb{R} \times \mathbb{R}^{d} \rightarrow \mathbb{R}$ and we assume that (1.1) holds for $(t, x)$ in a cylinder ${ }^{1}$, say

$$
P_{0}:=(0,1) \times(-1,1)^{d} .
$$

Then for $R<\frac{1}{2}$ the $L^{\infty}$ norm $\|u\|_{P_{R}}$ of $u$ on the unit cylinder minus a parabolic boundary layer of size $R$

$$
P_{R}:=\left(R^{2}, 1\right) \times(-(1-R), 1-R)^{d},
$$

satisfies a bound which only depends on $R$ and a distributional norm of $\zeta$ restricted to the original cylinder $P_{0}$ :

$$
\|u\|_{P_{R}} \leqslant C(\alpha, d, m) \max \left\{R^{-\frac{2}{m-1}},[\zeta]_{\alpha-2, P_{0}}^{\frac{2}{2+(m-1) \alpha}},\|g\|^{\frac{1}{m}}\right\}
$$

where $[\zeta]_{\alpha-2, P_{0}}$ the space-time Hölder norm of order $\alpha-2$ on $P_{0}$ (see (3.8) below for a precise definition), and $\|g\|$ refers to the supremum norm of $g$.

One possible application of the bound (1.3) is the construction of solutions to (1.1) on the full space. The standard approach to solve stochastic reaction diffusion equations $[21,10,8]$ consists of writing the equation in its mild form and solving the corresponding fixed point problem using Picard iterations. However, this approach requires a pathwise uniform-in- $x$ control on $\zeta$, which typically only holds on bounded domains or if $\zeta$ decays at $\infty$; the interesting case of spatially stationary noise cannot be treated directly in this way. This problem was overcome in [12] where solutions where first constructed on a sequence of growing tori and then a compactness argument in a space with weights was used to pass to the limit. The strong localisation obtained in (1.3) should allow to significantly simplify this construction.

The estimate (1.3) also has an interesting consequence for the stochastic integrability of $u$. In fact, we are mostly interested in the case where $\zeta$ is a random distribution with Gaussian tails such that $\mathbb{E}\left[e^{\epsilon[\zeta]_{\alpha-2, P_{0}}^{2}}\right]$ is finite for $\epsilon>0$ small enough. The estimate (1.3) then immediately implies that for any $R>0$ and for $\epsilon>0$ small enough we get $\mathbb{E}\left[e^{\epsilon\|u\|_{P_{R}}^{2+(m-1) \alpha}}\right]<\infty$. So $\|u\|_{P_{R}}$ has lighter tails than Gaussian. We observe that better pathwise regularity for $\zeta$ leads to better integrability with respect to the probability distribution for $u$. In the special case of one-dimensional reaction-diffusion equations where $\zeta$ is a space-time white noise, equation (1.1) equipped with suitable boundary conditions defines a reversible Markov process, and an explicit expression of the equilibrium measure is available. In Section 7 we argue that in this case the integrability

\footnotetext{
${ }^{1}$ Of course the equation (1.1) has to be interpreted in a distributional sense, so this condition means that it holds when tested against smooth test-functions which are supported in the cylinder, see (3.1).
} 
we derive from estimate (1.3) coincides with the integrability derived from the explicit invariant measure.

Finally, our method offers a new perspective on singular SPDE. Our starting point is Hairer's notion [11] of subcriticality which in the context of (1.1) states, roughly speaking, that the small scale behaviour of solutions should be determined by the interplay of the heat operator and the rough driving noise $\zeta$, while on large scales the non-linearity becomes dominant. We implement this philosophy by regularising (1.1) on a length scale $L$ by convolving the equation with a suitable regularising kernel, arriving at

$$
\left(\partial_{t}-\Delta\right) u_{L}=-u_{L}\left|u_{L}\right|^{m-1}+g(u)_{L}+\zeta_{L}+\left[u_{L}\left|u_{L}\right|^{m-1}-\left(u|u|^{m-1}\right)_{L}\right],
$$

where the subscript $L$ denotes a regularised quantity. The extra term $\left[u_{L}\left|u_{L}\right|^{m-1}-\right.$ $\left.\left(u|u|^{m-1}\right)_{L}\right]$ on the right hand side appears because regularisation and application of the polynomial do not commute. We then use a low regularity version of classical Schauder theory, Lemma B.1, to control the error term $\left[u_{L}\left|u_{L}\right|^{m-1}-\left(u|u|^{m-1}\right)_{L}\right]$. Using this bound, the remaining terms can be treated as in the smooth case (see Theorem 4.4).

The theory of regularity structures is indeed a main motivation for this work. A priori including the "coming-down from infinitiy" property have been proven for singular SPDEs, namely the dynamic $\phi_{2}^{2 m}[16,20]$ and $\phi_{3}^{4}$ models $[15,2,9]$ both on compact domains and on the full space. The works on $\phi_{3}^{4}$ all relied on Fourier methods, the method of paracontrolled distributions, rather than the theory of regularity structures. The bounds obtained there imply coming down from infinity in time only, in the case of $\phi_{3}^{4}$ on the full space [9] in a weighted space. The ideas presented here can be extended to these more singular equations when the low regularity Schauder estimate, Lemma B.1, is replaced by a suitable version of the Schauder estimates from the theory of regularity structures. This is the content of our companion paper [14]. There we show that our method significantly simplifes the technical arguments used in [15, 2, 9] and extend its scope to construct solutions on the full space without the need for weights.

In the more regular case presented here it would be natural to aim to also include more general non-linearities, such as functions with faster than polynomial growth (e.g. the $\exp (\phi)$ model $f(u)=\sinh (u)$, see [1]) or functions of slower than polynomial growth such as $f(u) \sim u \log (u)^{\delta}$ for $\delta>2$. In this case the commutator term arising in (1.4) turns into

$$
f\left(u_{L}\right)-f(u)_{L} .
$$

Unfortunately, our method crucially on the fact that $x f^{\prime}(x) \lesssim f(x)$ which holds for polynomial $f$, but not for functions with exponential growth. Also, another part of our argument excludes functions that grow to slow (in the proof of Theorem 3.1 we need to $\operatorname{sum} \Theta(u):=\frac{f(u)}{u}$ for $u=2^{-k}, k \in \mathbb{N}$ ), thus essentially restricting us to polynomial $f$. However, in the case of a more general non-linearity $f$, we implement a more standard argument based on subtracting the solution $w$ to the linear equation

$$
\left(\partial_{t}-\Delta\right) w=\zeta
$$

and we do not pass through the regularised equation (1.4). We then get the property of "coming down from infinity" for the remainder $u-w$ in Corollary 4.6. For example, when $f(u)=\sinh (u)$, the strong damping implies that $u$ "comes down from infinity" much more quickly than in the polynomial case - in this case the function $R^{-\frac{2}{m-1}}$ in (1.3) turns into $\Theta^{-1}\left(R^{-2}\right)$, where $\Theta(R)=\frac{\sinh (R)}{R}$. For very weak damping $f(u) \sim u \log (u)^{\alpha+2}$, we obtain a slow coming down from infinity, of $\operatorname{order} \exp \left(R^{-\frac{2}{\alpha}}\right)$. In fact, this method is even easier than the method we use for the polynomial case, but it has two significant disadvantages: On the one hand, it is impossible to measure the fine interplay between regularity of $\zeta$ and integrability of $u$ in this way, because the remainder $u-w$ can never have better 
integrability than the Gaussian process $w$. More importantly, the more sophisticated method we use in the proof of our main theorem is crucial when dealing with more singular equations [14].

The rest of the paper is structured as follows: In Section 2 we discuss the elementary case of the stochastic ODE

$$
d x(t)=-|x(t)|^{m-1} x(t) d t+d w(t),
$$

in which our strategy and also the interplay between the regularity of the noise and integrability of the solution becomes apparent in a technically simple context. In Section 3 we introduce the framework and state the main result. The proof is split into Sections 4-5: In Section 4 we present a proof of the "space-time coming down from infinity" in the case where $\zeta$ is replaced by a smooth function. The argument relies on a maximum principle. As a Corollary, as discussed above, we derive the bounds on the remainder $u-w$ in the case of general, not necessarily polynomial $f$. In Section 5 the result of Section 4 is applied to the regularised equation 1.4 and combined with Schauder estimates to bound the commutator concluding the proof of our main result. In Section 6 we discuss the case of a random distribution $\zeta$ given by the time-derivative of the stochastic integral $\int_{0}^{t} \sigma d W$ for an adapted bounded process $\sigma=\sigma(s, x)$ and a distribution valued Wiener process $W$ with suitable (spatial) covariance operator. We show Gaussian estimates for $[\zeta]_{\alpha-2}$ and thus better than Gaussian bounds for $u$. Finally, in the special case of space-time white noise in one spatial dimension we show that the integrability obtained from our method coincides with the integrability of the process in equilibrium obtained from the explicit invariant measure.

\section{The ODE case}

Before dealing with equation (1.1) we briefly discuss the case of a (stochastic) ordinary differential equation

$$
d x(t)=-|x(t)|^{m-1} x(t) d t+d w(t)
$$

for a standard Brownian motion $w(t)$ and for $m>1$. It is well known that (2.1) defines a reversible Markov process with respect to the measure

$$
\mu(d x) \propto \exp \left(-\frac{2}{m+1}|x|^{m+1}\right) d x .
$$

We seek to derive optimal bounds on solutions of $x(t)$ directly from the equation (2.1).

As a starting point, consider the case of an ordinary differential equation driven by a regular noise term $\eta$

$$
\dot{x}(t)=-x(t)|x(t)|^{m-1}+\eta(t) .
$$

A simple ODE comparison Lemma, see [20, Lemma 3.8], shows that for $t \in(0,1]$

$$
|x(t)| \leqslant C(m) \max \left\{t^{-\frac{1}{m-1}},\left(\sup _{t \in[0,1]}|\eta(t)|\right)^{\frac{1}{m}}\right\},
$$

uniformly over all choices of initial datum $x(0)$. If $\eta$ is a Gaussian process, such that the random variable $\sup _{t \in[0,1]}|\eta(t)|$ has finite Gaussian moments, this bound implies that for $\epsilon>0$ small enough

$$
\mathbb{E}\left[\exp \left(\epsilon|x(1)|^{2 m}\right)\right]<\infty .
$$

In particular, in this regular case we get much better integrability than under the measure (2.2). The following deterministic lemma shows that the difference in integrability is closely related to the regularity of the driving signal. 
As usual, we define the $\alpha$-Hölder semi-norm as

$$
[w]_{\alpha}=\sup _{0 \leqslant s<t \leqslant 1} \frac{|w(t)-w(s)|}{|t-s|^{\alpha}} .
$$

In an attempt to make this proof as similar as possible to the one of our main result, Theorem 3.1, we relabel the regularity parameter as $\frac{\alpha}{2}$ for $\alpha \in(0,2)$.

Lemma 2.1. Let $w:[0,1] \rightarrow \mathbb{R}$ be $\frac{\alpha}{2}$-Hölder continuous for some $\alpha \in(0,2)$ with $w(0)=0$. For some $m>1$ let $x:[0,1] \rightarrow \mathbb{R}$ be a continuous solution to

$$
x(t)=x(0)-\int_{0}^{t}|x(s)|^{m-1} x(s) d s+w(t) .
$$

Then for $t \in(0,1]$

$$
|x(t)| \lesssim \max \left\{t^{-\frac{1}{m-1}},[w]_{\frac{\alpha}{2}}^{\frac{2}{2+(m-1) \alpha}}\right\} .
$$

Here and in the proof we use the symbol $\lesssim$ for $\leqslant C(\alpha, m)$.

If $w$ is a random function for which $[w]_{\alpha}$ has Gaussian tails this estimate yields

$$
\mathbb{E}\left[\exp \left(\epsilon|x(1)|^{2+(m-1) \alpha}\right)\right]<\infty,
$$

for $\epsilon$ small enough. In the Brownian case where $\alpha=1$ - the exponent $2+(m-1) \alpha$ becomes $1+m$ - in line with (2.2) and as $\alpha$ approaches 2 , the exponent becomes $2 m$ in line with (2.5).

Proof of Lemma 2.1. The proof follows the same steps, as the proof of the PDE result, Theorem 3.1, even though most are considerably simpler.

Step 1: Local Schauder estimate.

In this context a "Schauder estimate" is trivially derived, simply by writing for $0<t_{1}<$ $t_{2}<1$

$$
\begin{aligned}
\left|x\left(t_{2}\right)-x\left(t_{1}\right)\right| & =\left.\left|\int_{t_{1}}^{t_{2}} x(s)\right| x(s)\right|^{m-1} d s+w\left(t_{2}\right)-w\left(t_{1}\right) \mid \\
& \leqslant\left|t_{2}-t_{1}\right|\|x\|_{\left[t_{1}, t_{2}\right]}^{m}+\left|w\left(t_{2}\right)-w\left(t_{1}\right)\right|,
\end{aligned}
$$

which can be restated as

$$
[x]_{\frac{\alpha}{2},(s-L, s)} \leqslant L^{1-\frac{\alpha}{2}}\|x\|_{(s-L, s)}^{m}+[w]_{\frac{\alpha}{2}} .
$$

\section{Step 2: Application of a comparison lemma.}

We regularise equation (2.6) by convolution. To this end we introduce a smooth nonnegative kernel $\Psi: \mathbb{R} \rightarrow \mathbb{R}$ which is compactly supported in $[0,1]$ with $\int \Psi=1$ and set $\Psi_{L}(t)=\frac{1}{L} \Psi\left(\frac{t}{L}\right)$. For any function $f:(0,1) \rightarrow \mathbb{R}$ and for $t \in(L, 1)$ we define the regularisation $f_{L}(t)=f * \Psi_{L}(t)=\int_{t-L}^{t} \Psi_{L}(t-s) f(s) d s$.

Convolving the integral equation (2.6) with $\Psi_{L}$ and taking a time derivative leads to

$$
\dot{x}_{L}(t)=-x_{L}(t)\left|x_{L}(t)\right|^{m-1}+\dot{w}_{L}(t)+\left[\cdot^{m},(\cdot)_{L}\right] x(t) \quad \text { for } t \in(L, 1),
$$

where we write $\left[\cdot^{m},(\cdot)_{L}\right] x=\left[x_{L}\left|x_{L}\right|^{m-1}-\left(x|x|^{m-1}\right)_{L}\right]$ for the commutator term on the right hand side.

Now we can apply the ODE comparison result (2.4) to get, for all $t \in(L, 1]$

$$
\left|x_{L}(t)\right| \lesssim \max \left\{(t-L)^{-\frac{1}{m-1}},\left(\sup _{[L, 1]}\left|\dot{w}_{L}\right|\right)^{\frac{1}{m}},\left(\sup _{[L, 1]}\left|\left[\cdot^{m},(\cdot)_{L}\right] x\right|\right)^{\frac{1}{m}}\right\} .
$$


Step 3: Bound on the commutator.

To replace $x_{L}$ by $x$ and to bound the commutator term on the right hand side, we use the information on the regularity of $x$ provided by Step 1 . Indeed, using the fact that $\Psi$ has integral 1, we first see for $t \in(L, 1]$,

$$
\begin{aligned}
\left|\left(x_{L}-x\right)(t)\right| & =\left|\int_{t-L}^{t} \Psi_{L}(t-s)(x(s)-x(t)) d s\right| \\
& \leqslant[x]_{\frac{\alpha}{2},(t-L, t)} \int_{t-L}^{t} \Psi_{L}(t-s)|s-t|^{\frac{\alpha}{2}} d s \leqslant L^{\frac{\alpha}{2}}[x]_{\frac{\alpha}{2},(t-L, t)},
\end{aligned}
$$

where $[x]_{\frac{\alpha}{2}, I}=\sup _{s \neq t \in I} \frac{|x(t)-x(s)|}{|t-s|^{\frac{\alpha}{2}}}$ denotes the $\frac{\alpha}{2}$-Hölder semi-norm of $x$ restricted to the interval $I$. Similarly we establish a bound on the commutator: for $s \geqslant L$,

$$
\left|\left[\cdot^{m},(\cdot)_{L}\right] x(s)\right| \lesssim\|x\|_{(s-L, s)}^{m-1} L^{\frac{\alpha}{2}}[x]_{\frac{\alpha}{2},(s-L, s)},
$$

where $\|x\|_{I}$ is the supremum norm of $x$ restricted to the interval $I$. To see (2.12) we first write

$$
\left|\left[\cdot^{m},(\cdot)_{L}\right] x(s)\right|=\left|\int_{s-L}^{s} \Psi_{L}(s-r)\left(x_{L}(s)\left|x_{L}(s)\right|^{m-1}-x(r)|x(r)|^{m-1}\right) d r\right| .
$$

Then, using the mean value theorem and $\left|x_{L}(s)\right| \leqslant\|x\|_{(s-L, s)}$, we have

$$
\left.\left|x_{L}(s)\right| x_{L}(s)\right|^{m-1}-x(r)|x(r)|^{m-1}\left|\leqslant m\|x\|_{(s-L, s)}^{m-1}\right| x_{L}(s)-x(r) \mid .
$$

Finally, using the triangle inequality in the form $\left|x_{L}(s)-x(r)\right| \leqslant\left|x_{L}(s)-x(s)\right|+\mid x(s)-$ $x(r) \mid \leqslant 2 L^{\frac{\alpha}{2}}[x]_{\frac{\alpha}{2},(s-L, s)}$, we arrive at (2.12).

\section{Step 4: Post processing.}

Concerning the noise term on the right hand side of (2.10) we write

$$
\sup _{t \in[L, 1]}\left|\dot{w}_{L}(t)\right|=\sup _{t \in[L, 1]}\left|\int_{t-L}^{t} \dot{\Psi}_{L}(t-s)(w(s)-w(t)) d s\right| \lesssim L^{\frac{\alpha}{2}-1}[w]_{\frac{\alpha}{2}} .
$$

Combining (2.10), (2.12), (2.8) and (2.13) we arrive at

$$
\begin{aligned}
\left|x_{L}(t)\right| \lesssim \max \{ & (t-L)^{-\frac{1}{m-1}},\left(L^{\frac{\alpha}{2}-1}[w]_{\frac{\alpha}{2}}\right)^{\frac{1}{m}},\left(L\|x\|_{(t-L, t)}^{2 m-1}\right)^{\frac{1}{m}}, \\
& \left.\left.\left(L^{\frac{\alpha}{2}}\|x\|_{(t-L, t)}^{m-1}[w]_{\frac{\alpha}{2}}^{m}\right]\right)^{\frac{1}{m}}\right\}, \quad t>L .
\end{aligned}
$$

Combining this estimate with (2.11) and (2.8) this estimate turns into

$$
\begin{aligned}
|x(t)| \lesssim \max \{ & (t-L)^{-\frac{1}{m-1}},\left(L^{\frac{\alpha}{2}-1}[w]_{\frac{\alpha}{2}}\right)^{\frac{1}{m}},\left(L\|x\|_{(t-L, t)}^{2 m-1}\right)^{\frac{1}{m}}, \\
& \left.\left.\left(L^{\frac{\alpha}{2}}\|x\|_{(t-L, t)}^{m-1}[w]_{\frac{\alpha}{2}}\right]\right)^{\frac{1}{m}}, L\|x\|^{m}, L^{\frac{\alpha}{2}}[w]_{\frac{\alpha}{2}}\right\}, \quad t>L .
\end{aligned}
$$

Step 5: Choosing $L$.

We choose $L=\frac{\mu}{\|x\|_{(0,1)}^{m-1}}$ for $\mu=\mu(\alpha, m)>0$ small enough and consider $t$ satisfying $(t-L)^{-\frac{1}{m-1}} \leqslant \frac{1}{2}\|x\|_{(0,1)}$. Then applying Young's inequality $x y \leqslant \delta x^{p}+C(\delta) y^{p^{\prime}}$ for $\delta>0$ and $p, p^{\prime} \in(0,1)$ with $\frac{1}{p}+\frac{1}{p^{\prime}}=1$ multiple times yields

$$
\|x\|_{\left(\left(2^{m-1}+\mu\right)\|x\|_{(0,1)}^{1-m}, 1\right)} \leqslant \max \left\{\frac{1}{2}\|x\|_{(0,1)}, C[w]_{\frac{\alpha}{2}}^{\frac{2}{2+(m-1) \alpha}}\right\},
$$

for some constant $C=C(\alpha, m)$. Note that we can assume that $\left(2^{m-1}+\mu\right)\|x\|_{(0,1)}^{1-m}<1$, because else we trivially have a bound on $\|x\|_{(0,1)}$. 


\section{Step 6: Iterating the result.}

We now define a finite set $0=t_{0}<\ldots<t_{N}=1$ by setting $t_{n+1}-t_{n}=\left(2^{m-1}+\right.$ $\mu)\|x\|_{\left(t_{n}, 1\right)}^{1-m}$ as long as the time $t_{n+1}$ defined this way stays strictly less than 1 . We terminate the sequence, once this algorithm would produce a $t_{n+1} \geq 1 \mathrm{in}$ which case we set $t_{n+1}=t_{N}=1$. Note that $\left(2^{m-1}+\mu\right)\|x\|_{\left(t_{n}, 1\right)}^{1-m}$ is increasing in $n$ so the sequence necessarily terminates after finitely many steps.

Applying (2.14) to the equation restarted at the times $t_{n}$ we obtain for $n \leqslant N-1$

$$
\|x\|_{\left(t_{n}, 1\right)} \leqslant \max \left\{\frac{1}{2}\|x\|_{\left(t_{n-1}, 1\right)}, C[w]_{\frac{\alpha}{2}}^{\frac{2}{2+(m-1) \alpha}}\right\} .
$$

We now show that for $n<N$

$$
\|x\|_{\left(t_{n}, 1\right)} \lesssim \max \left\{t_{n+1}^{-\frac{1}{m-1}},[w]_{\frac{\alpha}{2}}^{\frac{2}{2+(m-1) \alpha}}\right\} .
$$

When the maximum in (2.15) is realised by $C[w]_{\frac{\alpha}{2}}^{\frac{\alpha}{1+(m-1) \frac{\alpha}{2}}}$, then this follows immediately.

Else, we have for $k \leqslant n,\|x\|_{\left(t_{n}, 1\right)} \leqslant\|x\|_{\left(t_{k}, 1\right)} 2^{k-n}$ and hence

$$
\begin{aligned}
t_{n+1} & =\sum_{k=0}^{n} t_{k+1}-t_{k}=\left(2^{m-1}+\mu\right) \sum_{k=0}^{n}\|x\|_{\left(t_{k}, 1\right)}^{1-m} \\
& \leqslant\left(2^{m-1}+\mu\right)\|x\|_{\left(t_{n}, 1\right)}^{1-m} \sum_{k=0}^{n} 2^{(n-1-k)(1-m)} \lesssim\|x\|_{\left(t_{n}, 1\right)}^{1-m},
\end{aligned}
$$

establishing (2.16). For the end point $t_{N}$ we have either $t_{N-1} \geqslant \frac{1}{2}$ or $t_{N}-t_{N-1} \geqslant \frac{1}{2}$. In the first case we invoke (2.17) for $n=N-1$ and in the second case the definition of $t_{n+1}-t_{n}$, in both cases yielding the existence of a constant $C$ such that

$$
\|x\|_{\left(t_{N-1}, 1\right)}^{1-m} \geqslant C \Rightarrow\|x\|_{\left(t_{N-1}, 1\right)} \leqslant C^{\frac{1}{1-m}},
$$

so (2.16) also holds for $n=N$. Finally, for intermediate points $t \in\left[t_{n-1}, t_{n}\right]$ we write

$$
\begin{aligned}
|x(t)| \leqslant\|x\|_{\left(t_{n-1}, 1\right)} & \stackrel{(2.16)}{\lesssim} \max \left\{t_{n}^{-\frac{1}{m-1}},[w]_{\frac{\alpha}{2}}^{\frac{2}{2+(m-1) \alpha}}\right\} \\
& \leqslant \max \left\{t^{-\frac{1}{m-1}},[w]_{\frac{\alpha}{2}}^{\frac{\alpha}{2+(m-1) \alpha}}\right\},
\end{aligned}
$$

so (2.7) follows.

\section{Setting and main result}

After this short interlude, we now go back to the parabolic equation (1.1). Throughout the rest of the paper we will say a continuous function $u$ satisfies (1.1) on an open set $B \subseteq \mathbb{R}_{t} \times \mathbb{R}_{x}^{d}$ if for all smooth functions $\eta$ which are supported in $B$ we have

$$
\int u\left(-\partial_{t}-\Delta\right) \eta=-\int f(u) \eta+\int \zeta \eta,
$$

where the last integral $\int \zeta \eta$ should be interpreted as the duality pairing between a distribution and a test function. As usual when dealing with parabolic equations, regularity will be measured with respect to the metric

$$
d((t, x),(\bar{t}, \bar{x}))=\max \{|x-\bar{x}|, \sqrt{|t-\bar{t}|}\},
$$


where $|\cdot|$ denotes the Euclidean norm on $\mathbb{R}^{d}$. We introduce the parabolic ball of center $z=(x, t)$ and radius $R$ in this metric $d$, looking only into the past:

$$
B(z, R)=\left\{\bar{z}=(\bar{t}, \bar{x}) \in \mathbb{R} \times \mathbb{R}^{d}, d(z, \bar{z})<R, \bar{t}<t\right\} .
$$

Recall that $P_{R}$ is the cylinder at distance $R$ from $P_{0}$, as introduced in (1.2). Note that for $R^{\prime}<R$ we have $P_{R^{\prime}}+B\left(0, R^{\prime}-R\right) \subset P_{R}$.

For $\alpha \in(0,1)$, we define the Hölder semi-norm [.] $]_{\alpha}$

$$
[u]_{\alpha}:=\sup _{z \neq \bar{z} \in \mathbb{R}^{\prime} \times \mathbb{R}^{d}} \frac{|u(z)-u(\bar{z})|}{d(z, \bar{z})^{\alpha}} .
$$

We will often deal with local quantities: If $B \subset \mathbb{R} \times \mathbb{R}^{d}$ is a bounded set, then we define the local $\alpha$-Hölder semi-norm [.] $]_{\alpha, B}$ as in (3.4) with the supremum restricted to $z, \bar{z} \in B$. Similarly, $\|\cdot\|$ denotes the supremum norm on the whole space $\mathbb{R} \times \mathbb{R}^{d}$ and $\|\cdot\|_{B}$ the supremum norm over $B$.

To measure distributions in negative Hölder spaces, we introduce a family of mollification operators $\left\{(.)_{L}\right\}$ which are consistent with the scaling given by the heat operator $(x, t)=\left(l \bar{x}, l^{2} \bar{t}\right)$. For this we fix a non-negative smooth function $\Psi$ with support in $-B(0,1)$ with $\Psi(z) \in[0,1]$ for all $z$ and with integral 1 and for $L \in(0,1]$ set $\Psi_{L}(x, t)=\frac{1}{L^{d+2}} \Psi\left(\frac{x}{L}, \frac{t}{L^{2}}\right)$. We define the operator $(\cdot)_{L}$ by convolution with $\Psi_{L}$, noting that for any $L,(\cdot)_{L}$ is a contraction on with respect to $\|\cdot\|$. We wish to keep track of the support of the relevant functions. Since $\Psi_{L}$ is compactly support in $-B(0, L)$,

$$
\left\|h_{L}\right\|_{K} \leqslant\|h\|_{K+B(0, L)}
$$

for any bounded set $K$. Furthermore, we mention the estimate

$$
\int\left|\Psi_{L}(x-y)\right| d(x, y)^{\alpha} d y \leqslant L^{\alpha},
$$

which, as in (2.11) above, immediately implies that for any $h \in C^{\alpha}$, and for any bounded set $K$, we have

$$
\left\|h_{L}-h\right\|_{K} \leqslant L^{\alpha} \sup _{z \in K}[h]_{\alpha, B(z, L)} .
$$

Finally, we define the local $C^{\alpha-2}$ semi-norm of a distribution $\zeta$ for $\alpha-2<0$ as

$$
[\zeta]_{\alpha-2, K}=\sup _{L \leqslant 1}\left\|(\zeta)_{L}\right\|_{K} L^{2-\alpha} .
$$

This is a localised version of the Besov norm of $B_{\infty, \infty}^{\alpha-2}$ as defined, for example in [3, Theorem 2.34]. Note that, $[\zeta]_{\alpha-2, K}$ depends only on the behaviour of the distribution $\zeta$ on the set $K+B(0,1)$ (i.e. if $\zeta$ and $\widetilde{\zeta}$ coincide when tested against test-functions supported in this set, then $[\zeta-\widetilde{\zeta}]_{\alpha-2, K}=0$ ). Multiplication with a smooth function is a continuous operation with respect to this norm. We have for any smooth and compactly supported function $\eta$

$$
[\eta \zeta]_{\alpha-2} \leqslant C(\eta)[\zeta]_{\alpha-2, \operatorname{supp}(\eta)} .
$$

Estimates of this type are classical and are typically proved by choosing a convenient mollifying kernel $\Psi_{L}$, see e.g. [18] for estimates based on kernels $\Psi_{L}$ satisfying a semigroup property in $L$, or [3, Section 2.4] for a proof in the language of Littlewood-Paley theory. We refer to [18, Lemma A3] for a proof that norms defined for different kernels are equivalent. More complicated bounds of this type are also essential in our companion paper [14] and are discussed there at length.

We now state our main result, to be proven in Section 5 . 
Theorem 3.1. Assume that $f(u)=u|u|^{m-1}+g(u)$ with $m \geqslant 1, g$ bounded and $\zeta$ is of regularity $\alpha-2$ for some $\alpha>0$ in the sense of (3.8). There exists a constant $C=C(\alpha, m, d)$ such that if $u$ is continuous and solves (1.1) on the cylinder $P_{0}$ in the sense of of (3.1) then for all $R \in\left(0, \frac{1}{2}\right)$,

$$
\|u\|_{P_{R}} \leqslant C \max \left\{R^{-\frac{2}{m-1}},[\zeta]_{\alpha-2, P_{0}}^{\frac{2}{2+(m-1) \alpha}},\|g\|^{\frac{1}{m}}\right\} .
$$

\section{Maximum principle}

\subsection{Assumptions and statement}

We prove a space-time version of "coming down from infinity" when there is no distribution of negative regularity involved, but we allow for a more general non-linearity. Let $u$ be a $C^{2}$ function defined for $z \in \mathbb{R} \times \mathbb{R}^{d}$, for which the following holds point-wise for $z \in P_{0}$ when $u(z)>0$ :

$$
\left(\partial_{t}-\Delta\right) u \leqslant-f(u)+g .
$$

Assumption 4.1. We make the following assumptions on $f$ and $g$ :

1. $g$ is a bounded function;

2. $f$ is $C^{2}$ and $f^{\prime \prime}(u) \geqslant 0$ for $u>0$;

3. there exists a constant $c>1$ such that $u f^{\prime}(u) \geqslant c f(u)$ for $u>0$.

Define $\Theta(u)=\frac{f(u)}{u}$. By (3), $\Theta$ is increasing for $u>0$.

Theorem 4.2. Let $u \in C^{\infty}$ satisfy (4.1) for functions $f$ and $g$ satisfying Assumption 4.1. There exist $\lambda=\lambda(d)>0$ and $C=C(c, d)$ such that the following point-wise bound on $u$ holds for all $(t, x) \in(0,1) \times(-1,1)^{d}$ :

$$
u(x, t) \leqslant C \max \left\{\Theta^{-1}\left(\frac{1}{\lambda^{2} \min \left\{t,\left(1-x_{i}\right)^{2},\left(1+x_{i}\right)^{2}, i=1 \ldots d\right\}}\right), f^{-1}(\|g\|)\right\} .
$$

Note that $\min \left\{t,\left(1-x_{i}\right)^{2},\left(1+x_{i}\right)^{2}, i=1 \ldots d\right\}$ is exactly the square of the distance to the boundary of $[0,1] \times[-1,1]^{d}$ in the parabolic metric. Since a similar bound can be obtained for $-u$ under a suitable symmetry assumption, this gives a bound on $\|u\|_{P_{R}}$, depending only on $R$.

The condition $u f^{\prime}(u) \geqslant c f(u)$ with $c>1$ is verified exactly for $f(u)=u|u|^{c-1}$, hence any function with at least polynomial growth is included in this theorem. For such monomials, $\Theta^{-1}$ becomes $x \mapsto x^{\frac{1}{c-1}}$. For functions with faster growth, the bound is going to be even stronger. However, some functions with super-linear but not polynomial growth are not included. For example $f(u)=u \log (1+u)^{\alpha}$ for $\alpha>0$. For this example, $\frac{u f^{\prime}(u)}{f(u)}=1+\frac{u \alpha}{(1+u) \log (1+u)} \rightarrow 1$ as $u \rightarrow \infty$, so point (3) in Assumption 4.1 is violated. We can still get a result in that case, under a slightly weaker, but also slightly more complicated set of assumptions:

Assumption 4.3. We make the following assumptions on $f$ and $g$ :

1. $g$ is a bounded function;

2. $f$ is $C^{2}$ and $u f^{\prime}(u) \geqslant f(u)$ and there exist two $C^{2}$ functions $f_{1}$ and $f_{2}$ such that $f=f_{1} f_{2}$;

3. $f_{1}^{\prime \prime} \geqslant 0$ for $u>0$;

4. $f_{2} \geqslant c>0$ for $u>0$ and

$$
f_{2}(u) \geqslant \max \left\{\frac{1}{\left(\frac{u f_{1}^{\prime}(u)}{f_{1}(u)}-1\right)^{2}}, \frac{1}{\frac{u f_{1}^{\prime}(u)}{f_{1}(u)}-1}\right\} .
$$

Define now $\Theta(u)=\frac{f_{1}(u)}{u}$. $\Theta$ is increasing for $u>0$ by condition (4). 
In the example where we want to take $f_{1}(u)=u \log (1+u)^{\alpha}$ for $\alpha>0$, one can easily check that in order to satisfy condition $(4), f_{2}$ should be $\left(\frac{1+u}{\alpha u}\right)^{2} \frac{\log (1+u)^{2}}{\alpha}$ and hence $f(u)=\frac{(1+u)^{2}}{\alpha^{2} u} \log (1+u)^{2+\alpha}$ and $\Theta^{-1}(x)=\exp \left(x^{\frac{1}{\alpha}}\right)-1$. Note that this condition is slightly more restrictive than the Osgood condition $\int_{1}^{\infty} \frac{1}{f(u)} d u<\infty$, which would be satisfied by the function with slightly slower growth $f(u)=u \log (1+u)^{1+\alpha}$ for $\alpha>0$. Still we believe that our condition may be sharp, and that the slightly stronger growth requirement is due to a genuine difference between the ODE and the PDE setting.

Theorem 4.4. Let $u \in C^{\infty}$ solve (4.1) for functions $f$ and $g$ satisfying Assumption 4.3. There exist $\lambda=\lambda(d)>0$ and $C=C(c, d)$ such that the following point-wise bound on $u$, holds for all $(t, x) \in(0,1) \times(-1,1)^{d}$ :

$$
u(x, t) \leqslant C \max \left\{\Theta^{-1}\left(\frac{1}{\lambda^{2} \min \left\{t,\left(1-x_{i}\right)^{2},\left(1+x_{i}\right)^{2}, i=1 \ldots d\right\}}\right), f^{-1}(\|g\|)\right\} .
$$

Theorem 4.2 is implied by Theorem 4.4 by choosing $f_{1}=f$ and $f_{2}=\frac{1}{(c-1)^{2}}$.

Remark 4.5. The fact that under these more general assumptions $\Theta$ is not simply defined by $f(u) / u$ but instead grows more slowly, is the reason why we do not get an equivalent of Theorem 3.1, in the case of slower than polynomial growth.

\subsection{Bound on the remainder}

A first corollary of this result is a "coming down from infinity" result for the singular equation (1.1) with general non linearity. In the manner of [6], we expand around the solution to the linear equation: let $w$ solve

$$
\left(\partial_{t}-\Delta\right) w=\zeta \quad \text { on } P_{0} .
$$

Below in Section 6 we construct $w$ as the solution on the whole space of the heat equation with a $\zeta$ cut-off outside of $P_{0}$, for which $\|w\|$ is bounded by $[\zeta]_{\alpha-2}$, but this particular choice is not essential. Define $v=u-w$. If $u$ is a solution to

$$
\left(\partial_{t}-\Delta\right) u(z)=-f(u)+g(u, z)+\zeta,
$$

where we assume that $f, g$ satisfies the Assumption 4.3 then $v$ is a solution to

$$
\left(\partial_{t}-\Delta\right) v(z)=-f(v+w)+g(v+w, z)
$$

on $P_{0}$. We now use the $w$-dependent decomposition $f(v+w)=\widetilde{f}(v, z)+\widetilde{g}(v, z)$ defined by

$$
\widetilde{f}(v, z)= \begin{cases}f(v+w) & \text { if }|v(z)| \geq 2|w(z)| \\ f\left(\frac{v}{2}\right) & \text { else, }\end{cases}
$$

and $\widetilde{g}(v, z)=f(v+w)-\widetilde{f}(v, z)$. Then, on the one hand by monotonicity of $f$ we have $\widetilde{f}(v, z) \geqslant f\left(\frac{v}{2}\right)$ and on the other hand $\|\widetilde{g}\| \leqslant f(3\|w\|)$. The Assumptions 4.3 are then satisfied with $\widetilde{f}$ and $g+\widetilde{g}$ and we can apply Theorem 4.4 to get a bound on $v$, and then the triangle inequality to get bounds on $u$. We have

$$
f^{-1}(\|g+\widetilde{g}\|) \leqslant f^{-1}(2\|g\|)+6\|w\| .
$$

A corollary of Theorem 4.4 is then:

Corollary 4.6. Assume $\zeta \in C^{\alpha-2}$ for some $\alpha>0$. If $u$ is solution to (1.1) and $w$ is solution to (4.5), then there exists constants $C=C(c, d, \alpha)$ and $\lambda=\lambda(d)$ such that

$$
\|u\|_{P_{R}} \leqslant C \max \left\{\Theta^{-1}\left((\lambda R)^{-2}\right), f^{-1}(2\|g\|),\|w\|\right\} .
$$


Keeping in mind the motivation of stochastic PDEs, where $\zeta$ is the white noise, the drawback of the expansion around the solution to the linear equation is that the integrability of $u$ that we get out of this result is at best the one of $w$. As we will see in Section 7, Theorem 4.4 allows for better estimates than this in the polynomial case.

\subsection{Proof of Theorem 4.4}

We split the proof of the theorem in two lemmas. Lemma 4.7 states conditions on a function $\eta$ that imply a bound on the product $u \eta$, and Lemma 4.8 gives a particular choice of $\eta$ that satisfies the conditions of Lemma 4.7 and implies Theorem 4.4.

Lemma 4.7. Let $\eta$ be a continuous function defined on $\mathbb{R}_{+} \times[-1,1]^{d}, C^{2}$ and strictly positive on the interior and such that $\eta=0$ on the boundary. Assume that $\eta$ satisfies the following inequalities:

$$
\frac{\left(\partial_{t}-\Delta\right) \eta}{\eta}+2 \frac{|\nabla \eta|^{2}}{\eta^{2}} \leqslant \frac{\eta}{2} f\left(\frac{1}{\eta}\right)
$$

and

$$
0 \leqslant \eta \leqslant \frac{1}{f^{-1}(\|g\|)}
$$

Then if $u$ solves (4.1) it satisfies the bound $u \eta \leqslant 2$.

Proof. Take $u$ satisfying (4.1). Either $u \eta$ attains its maximum on $[0,1] \times[-1,1]^{d}$ at some point $z_{0} \in(0,1] \times(-1,1)^{d}$, or it is non-positive, in which case $u \leqslant 0$ in $[0,1] \times\{|x| \leqslant 1\}$. Assuming this is not the case, we get that at the maximum point, $0=\nabla(u \eta)\left(z_{0}\right)$, i.e.

$$
\nabla u\left(z_{0}\right)=-\frac{\nabla \eta}{\eta} u\left(z_{0}\right)
$$

If $z_{0} \in\{1\} \times(-1,1)^{d}$, then $\partial_{t} \eta u\left(z_{0}\right) \geqslant 0$. Else, $\partial_{t} \eta u\left(z_{0}\right)=0$. Additionally, $\Delta \eta u\left(z_{0}\right) \leqslant 0$ and therefore at the maximum we have

$$
\begin{aligned}
& 0 \leqslant\left(\partial_{t}-\Delta\right)(u \eta)=\eta\left(\partial_{t}-\Delta\right) u+u\left(\partial_{t}-\Delta\right) \eta-2 \nabla u \cdot \nabla \eta \\
& \stackrel{(4.1) ;(4.10)}{\leqslant}-\eta(f(u)-g)+u\left(\left(\partial_{t}-\Delta\right) \eta+2 \frac{|\nabla \eta|^{2}}{\eta}\right) .
\end{aligned}
$$

Then we get from (4.8)

$$
\frac{f(u)}{u} \leqslant \frac{\eta}{2} f\left(\frac{1}{\eta}\right)+\frac{\|g\|}{u} \leqslant 2 \max \left\{\frac{\eta}{2} f\left(\frac{1}{\eta}\right), \frac{\|g\|}{u}\right\} .
$$

If the maximum is realised by the first term, then $\frac{f(u)}{u} \leqslant \eta f\left(\frac{1}{\eta}\right)$. Since $u f^{\prime}(u) \geqslant f(u)$, $u \mapsto \frac{f(u)}{u}$ is increasing, we have that at $z_{0}, u \eta \leqslant 1$. If the maximum is realised by the second term, then it has to be bigger than the first one:

$$
\frac{\eta}{2} f\left(\frac{1}{\eta}\right) \leqslant \frac{\|g\|}{u} \Rightarrow u \eta \leqslant 2 \frac{\|g\|}{f\left(\frac{1}{\eta}\right)} .
$$

We then have that at $z_{0}, u \eta \leqslant 2$ under the condition (4.9).

Thus proving a bound on $u$ reduces to choosing a suitable function $\eta$ satisfying the inequalities (4.8) and (4.9). Ideally, we would like to take $\eta$ directly as

$$
\eta(x, t)=\min \left\{\Theta^{-1}\left(\frac{1}{\lambda^{2} t}\right)^{-1}, \Theta^{-1}\left(\frac{1}{\lambda^{2}\left(1-x_{i}\right)^{2}}\right)^{-1}, i=1 \ldots d, f^{-1}(\|g\|)^{-1}\right\} .
$$


This almost works as the individual function within the min do satisfy (4.8). Indeed, for example $\eta(x, t)=\Theta^{-1}\left(\frac{1}{\lambda^{2} t}\right)^{-1}$ satisfies

$$
\partial_{t} \eta=\frac{\lambda^{2}}{\left(\lambda^{2} t\right)^{2}} \frac{1}{\Theta^{\prime} \circ \Theta^{-1}\left(\frac{1}{\lambda^{2} t}\right)} \eta^{2} .
$$

We use $v=\Theta^{-1}\left(\frac{1}{\lambda^{2} t}\right) \leqslant \frac{1}{\eta}$ and $f=f_{1} f_{2}$. Given that $\Theta^{\prime}(y)=\frac{f_{1}^{\prime}(y)}{y}-\frac{f_{1}(y)}{y^{2}}$, we get

$$
\frac{\partial_{t} \eta}{\eta^{2} f\left(\frac{1}{\eta}\right)} \leqslant \frac{\lambda^{2}}{f\left(\frac{1}{\eta}\right)} \frac{\Theta(v)^{2}}{\Theta^{\prime}(v)} \leqslant \frac{\lambda^{2}}{f_{1}(v) f_{2}(v)} \frac{\Theta(v)^{2}}{\Theta^{\prime}(v)}=\frac{\lambda^{2}}{f_{2}(v)} \frac{1}{\frac{v f_{1}^{\prime}(v)}{f_{1}(v)}-1} .
$$

Applying the condition (4.3) gives a bound on this, independent of $v$, establishing (4.8). Unfortunately, taking the min of these functions is not an admitted operation due to the discontinuity of the derivative at the points where we join two solutions (recall that while the minimum of two supersolutions is a supersolution the maximum of two supersolutions in general is not).

In the following Lemma we overcome this problem by replacing the non-smooth function $\min \left\{x_{1}, \ldots, x_{n}\right\}$ in the above definition of $\eta$ by the smooth function

$$
\frac{1}{x_{1}^{-1}+\ldots x_{n}^{-1}} \text {. }
$$

Lemma 4.8. For $z=(t, x) \in(0, \infty) \times(-1,1)$, we define

$$
\eta(x, t)=\frac{1}{\Theta^{-1}\left(\frac{1}{\lambda^{2} t}\right)+\sum_{i=1}^{d}\left(\Theta^{-1}\left(\frac{1}{\lambda^{2}\left(1+x_{i}\right)^{2}}\right)+\Theta^{-1}\left(\frac{1}{\lambda^{2}\left(1-x_{i}\right)^{2}}\right)\right)+f^{-1}(\|g\|)},
$$

where $\lambda=(28 d+1)^{-\frac{1}{2}}$ and we continuously extend with the value 0 on the boundary of the domain. Then $\eta$ satisfies (4.8) and (4.9) and

$$
\frac{1}{\eta} \leqslant(2 d+1) \Theta^{-1}\left(\frac{1}{\lambda^{2} \min _{i}\left\{t,\left(1+x_{i}\right)^{2},\left(1-x_{i}\right)^{2}\right\}}\right)+f^{-1}(\|g\|)
$$

This choice of $\eta$ guarantees a bound on $u$ that is related to the distance from the boundary of $[0,1] \times[-1,1]^{d}$, independently of the boundary conditions. Lemmas 4.7 and 4.8 imply directly Theorem 4.4 .

Proof. The condition (4.9) is obvious, and so is (4.14). We have a bit more:

$$
\begin{aligned}
(2 d+1) \Theta^{-1} & \left(\frac{1}{\lambda^{2} \min _{i}\left\{t,\left(1+x_{i}\right)^{2},\left(1-x_{i}\right)^{2}\right\}}\right) \\
& \geqslant \frac{1}{\eta}-f^{-1}(\|g\|) \geqslant \Theta^{-1}\left(\frac{1}{\lambda^{2} \min _{i}\left\{t,\left(1+x_{i}\right)^{2},\left(1-x_{i}\right)^{2}\right\}}\right) .
\end{aligned}
$$

We will now check (4.8). For the time variable, we have as in (4.12)

$$
\partial_{t} \eta=\frac{\lambda^{2}}{\left(\lambda^{2} t\right)^{2}} \frac{1}{\Theta^{\prime} \circ \Theta^{-1}\left(\frac{1}{\lambda^{2} t}\right)} \eta^{2},
$$

and we have already seen that this implies

$$
\frac{\partial_{t} \eta}{\eta^{2} f\left(\frac{1}{\eta}\right)} \leqslant \lambda^{2} .
$$


We now consider the spatial derivatives.

$$
\begin{gathered}
\partial_{i} \eta=\frac{1}{\lambda^{2}}\left(\frac{2}{\left(1+x_{i}\right)^{3}} \frac{1}{\Theta^{\prime} \circ \Theta^{-1}\left(\frac{1}{\lambda^{2}\left(1+x_{i}\right)^{2}}\right)}-\frac{2}{\left(1-x_{i}\right)^{3}} \frac{1}{\Theta^{\prime} \circ \Theta^{-1}\left(\frac{1}{\lambda^{2}\left(1-x_{i}\right)^{2}}\right)}\right) \eta^{2}, \\
\partial_{i}^{2} \eta=-\frac{1}{\lambda^{2}}\left(\frac{6}{\left(1+x_{i}\right)^{4}} \frac{1}{\Theta^{\prime} \circ \Theta^{-1}\left(\frac{1}{\lambda^{2}\left(1+x_{i}\right)^{2}}\right)}+\frac{6}{\left(1-x_{i}\right)^{4}} \frac{1}{\Theta^{\prime} \circ \Theta^{-1}\left(\frac{1}{\lambda^{2}\left(1-x_{i}\right)^{2}}\right)}\right) \eta^{2} \\
+\frac{1}{\lambda^{4}}\left(\frac{4}{\left(1+x_{i}\right)^{6}} \frac{\Theta^{\prime \prime} \circ \Theta^{-1}\left(\frac{1}{\lambda^{2}\left(1+x_{i}\right)^{2}}\right)}{\left(\Theta^{\prime} \circ \Theta^{-1}\left(\frac{1}{\lambda^{2}\left(1+x_{i}\right)^{2}}\right)\right)^{3}}+\frac{4}{\left(1-x_{i}\right)^{6}} \frac{\Theta^{\prime \prime} \circ \Theta^{-1}\left(\frac{1}{\lambda^{2}\left(1-x_{i}\right)^{2}}\right)}{\left(\Theta^{\prime} \circ \Theta^{-1}\left(\frac{1}{\lambda^{2}\left(1-x_{i}\right)^{2}}\right)\right)^{3}}\right) \eta^{2} \\
+\frac{2}{\lambda^{4}}\left(\frac{2}{\left(1+x_{i}\right)^{3}} \frac{2}{\Theta^{\prime} \circ \Theta^{-1}\left(\frac{1}{\lambda^{2}\left(1+x_{i}\right)^{2}}\right)}-\frac{1}{\left(1-x_{i}\right)^{3}} \frac{1}{\Theta^{\prime} \circ \Theta^{-1}\left(\frac{1}{\lambda^{2}\left(1-x_{i}\right)^{2}}\right)}\right)^{2} \eta^{3} .
\end{gathered}
$$

Note that the last line is equal to $\frac{\partial_{i} \eta}{\eta^{2}} 2 \eta \partial_{i} \eta=2 \frac{\left(\partial_{i} \eta\right)^{2}}{\eta}$, hence it will cancel when computing $-\partial_{i}^{2} \eta+2 \frac{\left(\partial_{i} \eta\right)^{2}}{\eta}$. For the remaining terms, we use $v_{1, i}=\Theta^{-1}\left(\frac{1}{\lambda^{2}\left(1+x_{i}\right)^{2}}\right)$ and $v_{2, i}=\Theta^{-1}\left(\frac{1}{\lambda^{2}\left(1-x_{i}\right)^{2}}\right)$ and we get:

$$
\begin{aligned}
\frac{1}{\lambda^{2} f\left(\frac{1}{\eta}\right)}\left(-\frac{\partial_{i}^{2} \eta}{\eta^{2}}+2 \frac{\left(\partial_{i} \eta\right)^{2}}{\eta^{3}}\right)= & \frac{6 \Theta\left(v_{1, i}\right)^{2}}{f\left(\frac{1}{\eta}\right)\left(\frac{f_{1}^{\prime}\left(v_{1, i}\right)}{v_{1, i}}-\frac{f_{1}\left(v_{1, i}\right)}{v_{1, i}^{2}}\right)}+\frac{6 \Theta\left(v_{2, i}\right)^{2}}{f\left(\frac{1}{\eta}\right)\left(\frac{f_{1}^{\prime}\left(v_{2, i}\right)}{v_{2, i}}-\frac{f_{1}\left(v_{2, i}\right)}{v_{2, i}^{2}}\right)} \\
& -\frac{4 \Theta\left(v_{1, i}\right)^{3}}{f\left(\frac{1}{\eta}\right)} \frac{\frac{f^{\prime \prime}\left(v_{1, i}\right)}{v_{1, i}}-\frac{2}{v_{1, i}}\left(\frac{f_{1}^{\prime}\left(v_{1, i}\right)}{v_{1, i}}-\frac{f_{1}\left(v_{1, i}\right)}{v_{1, i}^{2}}\right)}{\left(\frac{f_{1}^{\prime}\left(v_{1, i}\right)}{v_{1, i}}-\frac{f_{1}\left(v_{1, i}\right)}{v_{1, i}^{2}}\right)^{3}} \\
& -\frac{4 \Theta\left(v_{2, i}\right)^{3}}{f\left(\frac{1}{\eta}\right)} \frac{\frac{f^{\prime \prime}\left(v_{2, i}\right)}{v_{2, i}}-\frac{2}{v_{2, i}}\left(\frac{f_{1}^{\prime}\left(v_{2, i}\right)}{v_{2, i}}-\frac{f_{1}\left(v_{2, i}\right)}{v_{2, i}^{2}}\right)}{\left(\frac{f_{1}^{\prime}\left(v_{2, i}\right)}{v_{2, i}}-\frac{f_{1}\left(v_{2, i}\right)}{v_{2, i}^{2}}\right)^{3}} .
\end{aligned}
$$

Using that $f$ is increasing, the bound (4.15) and $f=f_{1} f_{2}$, we have that $f\left(\frac{1}{\eta}\right) \geqslant$ $f_{1}\left(v_{j, i}\right) f_{2}\left(v_{j, i}\right)$ for $j \in\{1,2\}$. We also know that $f_{2}^{\prime \prime}>0$, hence we get:

$$
\begin{aligned}
\frac{1}{f\left(\frac{1}{\eta}\right)}\left(-\frac{\partial_{i}^{2} \eta}{\eta^{2}}+2 \frac{\left(\partial_{i} \eta\right)^{2}}{\eta^{3}}\right) & \leqslant \frac{6 \lambda^{2}}{f_{2}\left(v_{1, i}\right)\left(\frac{f_{1}^{\prime}\left(v_{1, i}\right) v_{1, i}}{f_{1}\left(v_{1, i}\right)}-1\right)}+\frac{6 \lambda^{2}}{f_{2}\left(v_{2, i}\right)\left(\frac{f_{1}^{\prime}\left(v_{2, i}\right) v_{2, i}}{f_{1}\left(v_{2, i}\right)}-1\right)} \\
& +\frac{8 \lambda^{2}}{f_{2}\left(v_{1, i}\right)\left(\frac{f_{1}^{\prime}\left(v_{1, i}\right) v_{1, i}}{f_{1}\left(v_{1, i}\right)}-1\right)^{2}}+\frac{8 \lambda^{2}}{f_{2}\left(v_{2, i}\right)\left(\frac{f_{1}^{\prime}\left(v_{2, i}\right) v_{2, i}}{f_{1}\left(v_{2, i}\right)}-1\right)^{2}} .
\end{aligned}
$$

We conclude this proof by using the condition (4.3) and the value $\lambda=(28 d+1)^{-\frac{1}{2}}$.

\section{Proof of Theorem 3.1}

From now on, $f(u)=|u|^{m-1} u$. In particular, Theorem 4.2 holds with $\Theta^{-1}\left(R^{-2}\right)=$ $R^{-\frac{1}{m-1}}$. The proof relies on two arguments. The small scale oscillations are controlled via Schauder theory and the large scale behaviour through the maximum principle derived in Section 4, which applies only to regular objects. A connection between the two is established via the convolution of the equation with the kernel introduced in Section 3, which produces a commutator term. The technicality of the proof lies in balancing the contribution of the commutator and the contribution of the irregular noise.

Throughout the proof, $\lesssim$ will denote a bound up to a multiplicative constant which may change from line to line, but will only depend on $d, m$ and $\alpha$. We will also write $u^{m}$ as a short-hand for $u|u|^{m-1}$, as in the case when $m$ is an odd integer. 
Step 1: Local Schauder estimate We claim that for any $R>0$, for any $k>2$,

$$
\begin{aligned}
{[u]_{\alpha, B(z, R)} \lesssim \sup _{L \leqslant k R} L^{2-\alpha}\left\|\left(\mathbf{1}_{\{B(z, k R)\}}\left(\partial_{t}-\Delta\right) u\right)_{L}\right\|+(k R)^{-\alpha}\|u\|_{B(z, k R)} } \\
\leqslant(k R)^{2-\alpha}\|u\|_{B(z, k R)}^{m}+(k R)^{2-\alpha}\|g\|+[\zeta]_{\alpha-2, B(z, k R)} \\
\\
+(k R)^{-\alpha}\|u\|_{B(z, k R)} .
\end{aligned}
$$

We prove this estimate by applying a cut-off functions and using the low-regularity Schauder estimate given in Lemma B.1. By scaling and translation, it is enough to prove for some $C^{\alpha}$ function $U$,

$$
[U]_{\alpha, B\left(0, \frac{1}{2}\right)} \lesssim \sup _{L \leqslant 1} L^{2-\alpha}\left\|\left(\mathbf{1}_{\{B(0,1)\}}\left(\partial_{t}-\Delta\right) U\right)_{L}\right\|+\|U\|_{B(0,1)} .
$$

Indeed, since $[u]_{\alpha, B\left(0, \frac{1}{k}\right)} \leqslant[u]_{\alpha, B\left(0, \frac{1}{2}\right)}$, if we have (5.2), define $U(t, x)=u\left((k R)^{2}(t-\right.$ $\left.\left.t_{0}\right), k R\left(x-x_{0}\right)\right)$. Then

$$
\begin{gathered}
\|u\|_{B(z, k R)}=\|U\|_{B(0,1)}, \quad[u]_{\alpha, B(z, R)}=(k R)^{\alpha}[U]_{\alpha, B\left(0, \frac{1}{k}\right)} \\
\sup _{L \leqslant k R} L^{2-\alpha}\left\|\left(\mathbf{1}_{\{B(z, k R)\}}\left(\partial_{t}-\Delta\right) u\right)_{L}\right\|=\sup _{L \leqslant 1} L^{2-\alpha}\left\|\left(\partial_{t}-\Delta\right)\left(U \mathbf{1}_{\{B(0,1)\}}\right)_{L}\right\| .
\end{gathered}
$$

We proceed to prove (5.2). Let $\eta$ be a cut-off function, with value 1 on $B\left(0, \frac{1}{2}\right)$ and 0 on $B(0,1)^{C}$, and such that $\|\nabla \eta\| \leqslant 4$ and $\left\|\left(\Delta+\partial_{t}\right) \eta\right\| \leqslant 4$. Then

$$
\left(\partial_{t}-\Delta\right) U \eta=\eta\left(\partial_{t}-\Delta\right) U+U\left(\partial_{t}+\Delta\right) \eta-2 \nabla \cdot(U \nabla \eta) .
$$

By applying Lemma B.1 to Equation (5.3) we get that:

$$
[U \eta]_{\alpha} \lesssim \sup _{0<L<1} L^{2-\alpha}\left\|\left(\eta\left(\partial_{t}-\Delta\right) U+U\left(\partial_{t}+\Delta\right) \eta-2 \nabla \cdot(U \nabla \eta)\right)_{L}\right\| .
$$

We apply the triangle inequality and make use of (3.9) to bound each of these terms as follows.

$$
\begin{aligned}
&\left\|\left(\eta\left(\partial_{t}-\Delta\right) U\right)_{L}\right\| \lesssim\left\|\left(\mathbf{1}_{\{B(0,1)\}}\left(\partial_{t}-\Delta\right) U\right)_{L}\right\|, \\
&\left\|\left(U\left(\partial_{t}+\Delta\right) \eta\right)_{L}\right\| \leqslant\left\|U\left(\partial_{t}+\Delta\right) \eta\right\| \lesssim\|U\|_{B(0,1)}, \\
&\left\|(\nabla .(U \nabla \eta))_{L}\right\|=\sup _{z} \int(U \nabla \eta)(z-\bar{z}) \cdot \nabla \Psi_{L}(\bar{z}) d \bar{z} \\
& \leqslant\|U \nabla \eta\|\left\|\nabla \Psi_{L}\right\|_{L^{1}} \\
& \lesssim \frac{1}{L}\|U\|_{B(0,1)} .
\end{aligned}
$$

Since $\alpha<1$, we have

$$
\begin{array}{r}
\sup _{0<L<1} L^{2-\alpha}\left\|\left(\eta\left(\partial_{t}-\Delta\right) U+U\left(\partial_{t}+\Delta\right) \eta-2 \nabla \cdot(U \nabla \eta)\right)_{L}\right\| \\
\lesssim\left\|\left(\mathbf{1}_{\{B(0,1)\}}\left(\partial_{t}-\Delta\right) U\right)_{L}\right\|+\|U\|_{B(0,1)} .
\end{array}
$$

This concludes the proof of (5.2), hence the proof of (5.1).

Step 2: Application of the maximum principle We convolve the equation (1.1) with $\Psi_{L}$, where $L \in(0,1)$ will be specified later:

$$
\left(\partial_{t}-\Delta\right) u_{L}=-\left(u_{L}\right)^{m}+g_{L}+\zeta_{L}+\left(\left(u_{L}\right)^{m}-\left(u^{m}\right)_{L}\right) .
$$


The anti-symmetry of $u \mapsto u^{m}$ and Theorem 4.4 implies that for all $0<R^{\prime}<R<\frac{1}{2}$,

$$
\begin{aligned}
\left\|u_{L}\right\|_{P_{R}} \lesssim \max \{ & \left(\frac{1}{\left(R-R^{\prime}\right)^{2}}\right)^{\frac{1}{m-1}},\|g\|^{\frac{1}{m}},\left\|\zeta_{L}\right\|_{P_{R^{\prime}}}^{\frac{1}{m}}, \\
& \left.\left(\left\|\left(u_{L}\right)^{m}-\left(u^{m}\right)_{L}\right\|_{P_{R^{\prime}}}\right)^{\frac{1}{m}}\right\} .
\end{aligned}
$$

The goal is now to balance the commutator and the term with the noise. This will be done by choosing the parameter $L$ appropriately in Step 5 below.

Step 3: Bounds on the commutator We need estimates on the commutator $\left(u_{L}\right)^{m}-$ $\left(u^{m}\right)_{L}$. This is obtained as $u$ is $C^{\alpha}$, using the moment bounds (3.6) and (3.7).

$$
\begin{aligned}
& \left(\left(u_{L}\right)^{m}-\left(u^{m}\right)_{L}\right)(z)=\int \Psi_{L}(z-\bar{z})\left(u_{L}(z)^{m}-u(\bar{z})^{m}\right) d \bar{z} \\
& =\int \Psi_{L}(z-\bar{z}) \int_{0}^{1}\left((u)_{L}(z)-u(\bar{z})\right) m\left(\lambda(u)_{L}(z)+(1-\lambda) u(\bar{z})\right)^{m-1} d \lambda d \bar{z} \\
& \leqslant m\|u\|_{B(z, L)}^{m-1} \int \Psi_{L}(z-\bar{z})\left(u_{L}(z)-u(z)+u(z)-u(\bar{z})\right) d \bar{z} \\
& \leqslant m\|u\|_{B(z, L)}^{m-1} \int \Psi_{L}(z-\bar{z})\left(L^{\alpha}[u]_{\alpha, B(z, L)}+[u]_{\alpha, B(z, L)} d(z, \bar{z})^{\alpha}\right) d \bar{z} \\
& \leqslant 2 m\|u\|_{B(z, L)}^{m-1} L^{\alpha}[u]_{\alpha, B(z, L)} .
\end{aligned}
$$

Since this is true for all $z \in P_{R}$,

$$
\left\|\left(u_{L}\right)^{m}-\left(u^{m}\right)_{L}\right\|_{P_{R}} \leqslant 2 m\|u\|_{P_{R-L}-1}^{m-1} \sup _{z \in P_{R}}[u]_{\alpha, B(z, L)} L^{\alpha} .
$$

Using the local Schauder estimate (5.1) gives, for any $k>2$ :

$$
\begin{aligned}
\left\|\left(u_{L}\right)^{m}-\left(u^{m}\right)_{L}\right\|_{P_{R}} & \lesssim L^{2} k^{2-\alpha}\left(\|u\|_{P_{R-k L}}^{2 m-1}+\|u\|_{P_{R-k L}}^{m-1}\|g\|\right) \\
& +\|u\|_{P_{R-L}-1}^{m-1} \sup _{z \in P_{R}}[\zeta]_{\alpha-2, B(z, k R)} L^{\alpha}+k^{-\alpha}\|u\|_{P_{R-k L}}^{m} .
\end{aligned}
$$

Step 4: Boot-strapping We show here that for $k, L$ such that $2(k+1) L \leqslant 1$, with as before $k>2$, and for $1 \geqslant R \geqslant 2(k+1) L$, we have

$$
\begin{aligned}
\|u\|_{P_{R}} \lesssim \max \{ & R^{\frac{2}{1-m}},\|g\|^{\frac{1}{m}},\left([\zeta]_{\alpha-2, P_{0}} L^{\alpha-2}\right)^{\frac{1}{m}},\left(L^{2} k^{2-\alpha}\right)^{\frac{1}{m}}\|u\|_{P_{0}}^{2-\frac{1}{m}}, \\
& \left(L^{2} k^{2-\alpha}\right)^{\frac{1}{m}}\|u\|_{P_{0}}^{1-\frac{1}{m}}\|g\|^{\frac{1}{m}},\left(\|u\|_{P_{0}}^{m-1}[\zeta]_{\alpha-2, P_{0}} L^{\alpha}\right)^{\frac{1}{m}}, k^{-\frac{\alpha}{m}}\|u\|_{P_{0}} \\
& \left.L^{2} k^{2-\alpha}\|u\|_{P_{0}}^{m}, L^{2} k^{2-\alpha}\|g\|, L^{\alpha}[\zeta]_{\alpha-2, P_{0}}, k^{-\alpha}\|u\|_{P_{0}},\right\} .
\end{aligned}
$$

We need to be careful with the sets that are concerned by the norms since our different estimates always require a bit more space. We use the bound (3.7) and the Schauder estimate (5.1) with $L$ playing the role of $R$ :

$$
\begin{gathered}
\|u\|_{P_{R}} \leqslant\left\|u_{L}\right\|_{P_{R}}+L^{\alpha} \sup _{z \in P_{R}}[u]_{\alpha, B(z, L)} \\
\leqslant\left\|u_{L}\right\|_{P_{R-L}}+L^{2} k^{2-\alpha}\left(\|u\|_{P_{R-k L}}^{m}+\|g\|\right) \\
+L^{\alpha}[\zeta]_{\alpha-2, P_{R-k L}}+k^{-\alpha}\|u\|_{P_{R-k L}}
\end{gathered}
$$


Defining $r=k L$ allows to apply the bounds (5.6), (5.8) and (3.8). As $r \geqslant 0, P_{r} \subset P_{0}$.

$$
\begin{aligned}
&\left\|u_{L}\right\|_{P_{R-L}} \lesssim \max \left\{(R-(k+1) L)^{\frac{2}{1-m}},\|g\|^{\frac{1}{m}},\left\|\zeta_{L}\right\|_{P_{r}}^{\frac{1}{m}},\left\|\left(u_{L}\right)^{m}-\left(u^{m}\right)_{L}\right\|_{P_{r}}^{\frac{1}{m}}\right\} \\
& \lesssim \max \left\{(R-(k+1) L)^{\frac{2}{1-m}},\|g\|^{\frac{1}{m}},\left([\zeta]_{\alpha-2, P_{0}} L^{\alpha-2}\right)^{\frac{1}{m}},\left(L^{2} k^{2-\alpha}\right)^{\frac{1}{m}}\|u\|_{P_{0}}^{2-\frac{1}{m}}\right. \\
&\left.\left(L^{2} k^{2-\alpha}\right)^{\frac{1}{m}}\|u\|_{P_{0}}^{1-\frac{1}{m}}\|g\|^{\frac{1}{m}},\left(\|u\|_{P_{0}}^{m-1}[\zeta]_{\alpha-2, P_{0}} L^{\alpha}\right)^{\frac{1}{m}}, k^{-\frac{\alpha}{m}}\|u\|_{P_{0}}\right\} .
\end{aligned}
$$

If we start with $R \geqslant 2(k+1) L$ then $R-(k+1) L \geqslant \frac{R}{2}$. Putting together (5.11) and (5.10) gives (5.9).

Step 5: Choosing $L$ In order to balance the term containing $\zeta$ in (5.9), we see that we should assign the value $L=\frac{\mu}{\|u\|_{P_{0}}^{\frac{m-1}{2}}}$ for some $\mu \in(0,1)$ to be chosen. Note also that as $\mu \in(0,1),\left(\mu^{\alpha-2} \vee \mu^{\alpha}\right)=\mu^{\alpha-2}$. Furthermore, we impose $\mu^{2} k^{2-\alpha} \leqslant 1$. Consequently, (5.9) becomes

$$
\begin{aligned}
\|u\|_{P_{R}} \lesssim \max & \left\{R^{\frac{2}{1-m}},\left(1+\left(\mu^{2} k^{2-\alpha}\right)^{\frac{1}{m}}\right)\|g\|^{\frac{1}{m}},\left(\mu^{\alpha-2}\|u\|_{P_{0}}^{(m-1) \frac{2-\alpha}{2}}[\zeta]_{\alpha-2, P_{0}}\right)^{\frac{1}{m}},\right. \\
& \left(\left(\mu^{2} k^{2-\alpha} \vee k^{-\alpha}\right)^{\frac{1}{m}}+\left(\mu^{2} k^{2-\alpha} \vee k^{-\alpha}\right)\right)\|u\|_{P_{0}}, \\
& \left.\mu^{2} k^{2-\alpha}\|u\|_{P_{0}}^{1-m}\|g\|, \frac{\mu^{\alpha}}{\|u\|_{P_{0}}^{(m-1) \frac{\alpha}{2}}}[\zeta]_{\alpha-2, P_{0}}\right\} .
\end{aligned}
$$

Step 6: Identification of terms We claim that the bound above implies that there exists a positive constants $C$ such that:

$$
\|u\|_{P_{R}} \leqslant C \max \left\{R^{\frac{2}{1-m}},\|g\|^{\frac{1}{m}},[\zeta]_{\alpha-2, P_{0}}^{\frac{1}{1+(m-1) \frac{\alpha}{2}}}, \frac{1}{2 C}\|u\|_{P_{0}}\right\} .
$$

We need to interpolate some of the arguments of the maximum in (5.12) with arguments of our goal (5.13). The first two terms are already in the right form. For the next one, Young's inequality gives that for any $\gamma>0$,

$$
\left(\mu^{\alpha-2}\|u\|_{P_{0}}^{(m-1) \frac{2-\alpha}{2}}[\zeta]_{\alpha-2, P_{0}}\right)^{\frac{1}{m}} \lesssim \mu^{\frac{\alpha-2}{m}} \max \left\{\gamma\|u\|_{P_{0}}, \gamma^{\frac{\frac{\alpha-2}{2}}{m-1}+\alpha}[\zeta]_{\alpha-2, P_{0}}^{\frac{1}{1+\left(m-\frac{\alpha}{2}\right.}}\right\} .
$$

The next term is also in the right form, provided one chooses first $k$ large, and then $\mu$ small. The last two terms can not be dealt with with classical interpolation, since they involve negative powers of $\|u\|_{P_{0}}$. For the first one, we state that always one of the following is true, for any $\gamma>0$ :

$$
\|u\|_{P_{0}}^{-(m-1)}\|g\| \leqslant \gamma\|u\|_{P_{0}} \text { or }\|u\|_{P_{0}}^{m} \leqslant \frac{1}{\gamma}\|g\| .
$$

The first case gives the last argument of our objective for $\gamma$ small enough. The second case gives $\|u\|_{P_{R}} \leqslant\|u\|_{P_{0}} \leqslant\left(\frac{1}{\gamma}\|g\|\right)^{\frac{1}{m}}$. We proceed similarly for the last term. One of the following is always true:

$$
\mu^{\alpha}\|u\|_{P_{0}}^{-(m-1) \frac{\alpha}{2}}[\zeta]_{\alpha-2, P_{0}} \leqslant \mu^{\alpha} \gamma\|u\|_{P_{0}} \text { or }\|u\|_{P_{0}}^{1+(m-1) \frac{\alpha}{2}} \leqslant \frac{1}{\gamma}[\zeta]_{\alpha-2, P_{0}} .
$$

Once again the first case gives the last argument of our objective for $\mu^{\alpha} \gamma$ small enough, and the second case gives $\|u\|_{P_{R}} \leqslant\|u\|_{P_{0}} \leqslant\left(\frac{1}{\gamma}[\zeta]_{\alpha-2, P_{0}}\right)^{\frac{1}{1+(m-1) \frac{\alpha}{2}}}$. We can then choose $k$ large, $\mu$ and $\gamma$ small to get the desired constant $C$. 
Step 7: Iterating the result The last argument of the maximum (5.13) is greater than the first one for all $R$ such that

$$
R \leqslant R_{1}:=\left(\frac{1}{2 C}\|u\|_{P_{0}}\right)^{\frac{1-m}{2}} .
$$

Let us check that this is not in contradiction with $R_{1} \geqslant 2(k+1) L$. By defintion of $L$ and $R_{1}$,

$$
2(k+1) L=2(k+1) \frac{\mu}{\|u\|_{P_{0}}^{\frac{m-1}{2}}}=2(k+1) \mu(2 C)^{\frac{1-m}{2}} R_{1} \leqslant R_{1} \Leftrightarrow 2(k+1) \mu(2 C)^{\frac{1-m}{2}} \leqslant 1 .
$$

Since $C>1$ and $m>1$, it is enough to have $2(k+1) \mu \leqslant 1$. This can be done since $\mu$ is chosen after $k$.

From this point, the result (5.13) can be iterated to get bounds for smaller and smaller parabolic boxes.

$$
\|u\|_{P_{\left(R+R_{n-1}\right)}} \leqslant C \max \left\{\frac{1}{2 C}\|u\|_{P_{R_{n-1}}},\|g\|^{\frac{1}{m}},[\zeta]_{\alpha-2, P_{0}}^{\frac{1}{1+(m-1) \frac{\alpha}{2}}}, \frac{1}{R^{\frac{2}{m-1}}}\right\} .
$$

Define $R_{n}$ recursively by

$$
R_{n}-R_{n-1}=\left(\frac{1}{2 C}\|u\|_{P_{R_{n-1}}}\right)^{\frac{1-m}{2}}=\left(\frac{1}{2 C} \frac{\|u\|_{P_{0}}}{2^{n-1}}\right)^{\frac{1-m}{2}} .
$$

We conclude by summing those increments:

$$
\begin{gathered}
R_{n}=\sum_{k=1}^{n} R_{k}-R_{k-1}=\sum_{k=1}^{n}\left(\frac{1}{2 C} \frac{\|u\|_{P_{0}}}{2^{k-1}}\right)^{\frac{1-m}{2}} . \\
=\left(\frac{\|u\|_{P_{0}}}{2 C}\right)^{\frac{1-m}{2}} \sum_{k=0}^{n-1}\left(2^{\frac{1-m}{2}}\right)^{k} \lesssim\left(\frac{\|u\|_{P_{0}}}{2 C}\right)^{\frac{1-m}{2}}
\end{gathered}
$$

The same arguments as in the proof of Lemma 2.1 concludes the proof of Theorem 3.1.

\section{Mutiplicative noise}

We present one example of equation where our result applies. Let $\left(\Omega, \mathcal{F}, \mathcal{F}_{t}, \mathbb{P}\right)$ be a filtered probability space and let $\left(W(t, \eta), t \geq 0, \eta \in C_{0}^{\infty}\left(\mathbb{R}^{d}\right)\right)$ be a Brownian motion with spatial covariance operator $K$ on $\Omega$. We assume that $K$ is given by the convolution with a function with controlled blow-up near the origin, i.e.

$$
K \phi(x)=\int_{\mathbb{R}^{d}} K\left(x-x^{\prime}\right) \phi\left(x^{\prime}\right) d x^{\prime},
$$

for $K \in C^{\infty}\left(\mathbb{R}^{d} \backslash\{0\}\right)$ satisfying

$$
|K(x)| \leqslant \frac{1}{|x|^{\lambda}},
$$

for some $\lambda<2$. If $\lambda>1$ and $d=1$, we allow additionally for a Dirac mass in the origin, in which case (6.1) turns into

$$
K \phi(x)=\int_{\mathbb{R}} K\left(x-x^{\prime}\right) \phi\left(x^{\prime}\right) d x^{\prime}+\phi(x) .
$$


In other words $\left(W(t, \eta), t \geq 0, \eta \in C_{0}^{\infty}\left(\mathbb{R}^{d}\right)\right)$ is a centred Gaussian process with covariances given either by

$$
\mathbb{E} W(t, \phi) W\left(t^{\prime}, \phi^{\prime}\right)=\left(t \wedge t^{\prime}\right) \int_{\mathbb{R}^{d}} \int_{\mathbb{R}^{d}} \phi(x) K\left(x-x^{\prime}\right) \phi^{\prime}\left(x^{\prime}\right) d x d x^{\prime}
$$

or in the one-dimensional case

$$
\begin{aligned}
& \mathbb{E} W(t, \phi) W\left(t^{\prime}, \phi^{\prime}\right) \\
& \quad=\left(t \wedge t^{\prime}\right)\left[\int_{\mathbb{R}} \int_{\mathbb{R}} \phi(x) K\left(x-x^{\prime}\right) \phi^{\prime}\left(x^{\prime}\right) d x d x^{\prime}+\int_{\mathbb{R}} \phi(x) \phi^{\prime}(x) d x\right] .
\end{aligned}
$$

Let $\left(\sigma(t, x), t \geq 0, x \in \mathbb{R}^{d}\right)$ be a progressively measurable process, with a deterministic $L^{\infty}$ bound, without loss of generality $|\sigma(t, x)| \leqslant 1$. Let $u(t, x)$ be a continuous process which satisfies the SPDE

$$
d u=(\Delta u-f(u)+g(u)) d t+\sigma d W
$$

on $P_{0}$, with $f$ satisfying the Assumptions 4.3. More precisely, for all $\eta \in C^{\infty}\left(\mathbb{R} \times \mathbb{R}^{d}\right)$ compactly supported in $P_{0}$ we assume that the following holds almost surely:

$$
\begin{aligned}
\iint & u\left(-\partial_{t}-\Delta\right) \eta d t d x \\
= & \iint(-f(u, z)+g(u, z)) \eta d t d x+\iint \eta(x) \sigma(t, x) d x d W(t, x),
\end{aligned}
$$

where $\iint \eta(x) \sigma(t, x) d x d W(t, x)$ should be interpreted as a stochastic integral, as defined in [8, Chapter 4]. The following lemma shows that the results of our deterministic analysis are applicable to this stochastic case.

The previous results do not depend on the particular choice of convolution kernel $\Psi$. We apply it with $\widetilde{\Psi}$ defined as

$$
\widetilde{\Psi}=\Psi_{\frac{1}{2}} * \Psi_{\frac{1}{2}},
$$

where $\Psi$ is as defined in Section 3. It is clear that $\widetilde{\Psi}$ is still non-negative, smooth and compactly supported in $B(0,1)$. We still write $(\cdot)_{L}$ for the convolution with $\Psi_{L}$ but we define the $C^{\alpha-2}$ norm with respect to $\widetilde{\Psi}$

$$
[\zeta]_{\alpha-2, C}=\sup _{0<L \leqslant 1}\left\|\left(\zeta_{\frac{L}{2}}\right)_{\frac{L}{2}}\right\|_{C} L^{2-\alpha}
$$

Lemma 6.1. We define a family of random variables $\left(\zeta(\eta), \eta \in C_{0}^{\infty}\left(\mathbb{R} \times \mathbb{R}^{d}\right)\right)$ by

$$
\zeta(\eta)=\iint \eta(x) \sigma(t, x) d x d W(t, x) .
$$

Then there exists a random distribution $\widetilde{\zeta}$ on $\Omega$ which almost surely takes values in $C^{\alpha-2}$ for any $\alpha<\frac{2-\lambda}{2}$ and such that for $\epsilon>0$ small enough

$$
\mathbb{E}\left[\exp \left(\epsilon[\widetilde{\zeta}]_{\alpha-2, P_{0}}^{2}\right)\right]<\infty .
$$

Furthermore $\widetilde{\zeta}$ is a modification of $\zeta$ in the sense that for all $\eta \in C_{0}^{\infty}\left(\mathbb{R} \times \mathbb{R}^{d}\right)$ we have almost surely

$$
\widetilde{\zeta}(\eta)=\zeta(\eta)
$$

We have the following corollaries. 
Corollary 6.2. Let $u$ solve the SPDE (6.4) in the sense of (6.5) for $f$ and $g$ satisfying Assumption 4.3. Define $\Theta(u)=\frac{f_{1}(u)}{u}$. Then there exists $\epsilon_{0}=\epsilon_{0}(c, d, \alpha)>0$ such that for $0<\epsilon \leqslant \epsilon_{0}$,

$$
\mathbb{E}\left[\exp \left(\epsilon\left(\sup _{0<R \leqslant \frac{1}{2}} \frac{\|u\|_{P_{R}}}{\Theta^{-1}\left((\lambda R)^{-2}\right)}\right)^{2}\right)\right]<\infty
$$

Proof. Let $\rho$ be a cut-off outside $P_{0}$ supported on $P_{0}+B(0,1)$, and $w$ be the bounded solution to

$$
\left(\partial_{t}-\Delta\right) w=\rho \xi
$$

vanishing for $t<-2$. From Lemma B.2, $\|w\| \lesssim[\xi]_{\alpha-2}$. Using Corollary 4.6 and Lemma 6.1 finishes the proof.

Using Theorem 3.1 in the case $f(u)=u|u|^{m-1}$, we have the more optimal estimate as follows:

Corollary 6.3. Let $u$ solve the SPDE (6.4) in the sense of (6.5) where $f(u, z)=u|u|^{m-1}$ and $g$ is bounded. Then there exists $\epsilon_{0}=\epsilon_{0}(m, d, \alpha)>0$ such that for $0<\epsilon \leqslant \epsilon_{0}$,

$$
\mathbb{E}\left[\exp \left(\epsilon\left(\sup _{0<R \leqslant \frac{1}{2}} R^{\frac{2}{m-1}}\|u\|_{P_{R}}\right)^{2+(m-1) \alpha}\right)\right]<\infty .
$$

The proof of Lemma 6.1 relies on the following technical lemma.

Lemma 6.4. The supremum $\sup _{0<L \leqslant 1}\left\|\zeta_{L}\right\|_{P_{0}}^{2 p} L^{2 p(2-\alpha)}$ is bounded by the supremum over dyadic $L$ only,

$$
\sup _{0<L \leqslant 1}\left\|\zeta_{L}\right\|_{P_{0}}^{2 p} L^{2 p(2-\alpha)} \lesssim \sup _{L=2^{-k} \leqslant 1}\left\|\zeta_{L}\right\|_{P_{0}+B(0,1)}^{2 p} L^{2 p(2-\alpha)} .
$$

Proof. By splitting the interval $(0,1)$ into $\left[2^{-n}, 2^{-n+1}\right)$ for $n \geqslant 1$, it is enough to prove that uniformly in $n \geqslant 1$ and $\lambda \in(0,1)$

$$
\left\|\zeta_{2^{-n}(1+\lambda)}\right\| \lesssim 2^{-n(\alpha-2)} \sup _{L=2^{-k} \leqslant 1} L^{2-\alpha}\left\|\zeta_{L}\right\| .
$$

This is a direct consequence of Lemma A.1 applied with $\Upsilon=\Psi_{2^{-n}(1+\lambda)}$ and $\theta=2^{-m}$ for some integer $m$ large enough.

Proof of Lemma 6.1. This Lemma is a variant of [16, Lemma 9] and we refer the reader to this Lemma for the construction of a suitable modification of $\zeta$. Here we only show the exponential integrability bound (6.7), using a similar argument as in [18, Lemma 4.1]. Throughout this proof, $\lesssim$ denotes a bound up to a constant that depend only on the dimension.

In the expansion in series of the exponential, we can exchange expectation and sum:

$$
\mathbb{E}\left[\exp \left(\epsilon^{2} \sup _{0<L \leqslant 1}\left\|\left(\zeta_{\frac{L}{2}}\right)_{\frac{L}{2}}\right\|_{P_{0}}^{2} L^{2(2-\alpha)}\right)\right]=\sum_{p=0}^{\infty} \epsilon^{2 p} \frac{\mathbb{E}\left[\sup _{0 \leqslant L \leqslant 1}\left\|\left(\zeta_{\frac{L}{2}}\right)_{\frac{L}{2}}\right\|_{P_{0}}^{2 p} L^{2 p(2-\alpha)}\right]}{p !} .
$$

Applying Lemma 6.4, we can bound the supremum over all $L$ by the sum over dyadic $L$.

$$
\mathbb{E}\left[\sup _{0<L \leqslant 1}\left\|\left(\zeta_{\frac{L}{2}}\right)_{\frac{L}{2}}\right\|_{P_{0}}^{2 p} L^{2 p(2-\alpha)}\right] \leqslant \sum_{L=2^{-k} \leqslant \frac{1}{2}} \mathbb{E}\left[\left\|\left(\zeta_{L}\right)_{L}\right\|_{P_{0}+B(0,1)}^{2 p}\right] L^{2 p(2-\alpha)} .
$$

Young's inequality implies

$$
\left\|\left(\zeta_{L}\right)_{L}\right\|_{P_{0}+B(0,1)} \leqslant\left\|\zeta_{L}\right\|_{L^{q}, P_{0}+B(0,2)}\left\|\Psi_{L}\right\|_{L^{q^{\prime}}},
$$


where the subscript means that the $L^{q}$ norm of $\zeta_{L}$ is taken over $P_{0}+B(0,2)$ and where $q^{\prime}=\frac{q-1}{q}$. By scaling, $\left\|\Psi_{L}\right\|_{L^{q^{\prime}}} \lesssim L^{-\frac{d+2}{q}}$. We apply this with $q=2 p$.

$$
\begin{aligned}
\mathbb{E}\left[\left\|\left(\zeta_{L}\right)_{L}\right\|^{2 p}\right] & \lesssim \mathbb{E}\left[\left\|\zeta_{L}\right\|_{L^{2 p}, P_{0}+B(0,2)}^{2 p}\right] L^{-(d+2)} \\
& =\mathbb{E}\left[\int_{P_{0}+B(0,2)} \zeta_{L}(t, x)^{2 p} d t d x\right] L^{-(d+2)} \\
& \lesssim \sup _{z \in P_{0}+B(0,2)} \mathbb{E}\left[\zeta_{L}(z)^{2 p}\right] L^{-(d+2)} .
\end{aligned}
$$

We bound $\mathbb{E}\left[\zeta_{L}(z)^{2 p}\right]$ using the boundedness of $\sigma$. Without loss of generality, we show the computation for $z=(0,0)$. By the Burkholder-Davies-Gundy inequality,

$$
\begin{aligned}
\mathbb{E}\left[\zeta_{L}(0,0)^{2 p}\right] & =\mathbb{E}\left[\left(\int_{(0,1)} \int_{\mathbb{R}^{d}} \Psi_{L}(t, x) \sigma(t, x) d W(t, x)\right)^{2 p}\right] \\
& \lesssim p^{p}\left(\iiint \Psi_{L}(t, x) \Psi_{L}\left(t, x^{\prime}\right) \sigma(t, x) \sigma\left(t, x^{\prime}\right) K\left(x-x^{\prime}\right) d t d x d x^{\prime}\right)^{p} \\
& +\mathbf{1}_{\{d=1, \lambda>1\}} p^{p}\left(\iint \Psi_{L}(t, x)^{2} \sigma(t, x)^{2} d t d x\right)^{p} \\
& \lesssim p^{p}\left(L^{-\lambda-2}+\mathbf{1}_{\{d=1, \lambda>1\}} L^{-d-2}\right)^{p} \lesssim p^{p} L^{-p(\lambda+2)} .
\end{aligned}
$$

We get that $\mathbb{E}\left[\left\|(\zeta)_{L}\right\|^{2 p}\right] L^{2 p(2-\alpha)} \lesssim p^{p} L^{p(2-2 \alpha-\lambda)-(d+2)}$. Since $2-2 \alpha-\lambda>0$, for $p$ large enough,

$$
\sum_{L=2^{-k} \leqslant \frac{1}{2}} \mathbb{E}\left[\left\|\left(\zeta_{L}\right)_{L}\right\|^{2 p}\right] L^{2 p(2-\alpha)} \lesssim p^{p} \frac{1}{1-2^{-p(2-2 \alpha-\lambda)+(d+2)}} .
$$

By Stirling's formula, for $p$ large enough,

$$
\epsilon^{2 p} \frac{\mathbb{E}\left[\sup _{0 \leqslant L \leqslant 1}\left\|\left(\zeta_{\frac{L}{2}}\right)_{\frac{L}{2}}\right\|^{2 p} L^{2 p(2-\alpha)}\right]}{p !} \lesssim \epsilon^{2 p} e^{p} \sqrt{p}
$$

hence for $\epsilon<e^{-2}$, (6.7) is verified.

\section{Invariant measure and optimality}

In this last section, we consider a special case of the SPDE considered in Section 6, namely the case of a one-dimensional reaction-diffusion equation driven by an additive space-time white noise. We aim to argue that in this case the bound obtained in Corollary 6.3 is optimal in terms of stochastic integrability.

Let $d=1$ and let $W$ be as in Section 6 with covariance operator $K \eta(x)=\eta(x)$. It is well-known [7, Section 11.2] that if we impose Dirichlet boundary conditions on the space-interval $[-1,1]$, then (6.5) defines a reversible Markov process with respect to the measure

$$
\frac{1}{Z} \exp \left(-\int_{-1}^{1} \frac{1}{m+1}|u(x)|^{m+1} d x\right) \mu(d u)
$$

where $\mu$ is the law of an appropriately scaled Brownian bridge and $Z$ is a normalisation constant. From the explicit expression (7.1) one can immediately read off that under this measure the following expectations are finite for $\alpha<\frac{1}{2}$ and $\epsilon$ small enough

$$
\mathbb{E}\left[\exp \left(\epsilon \int_{-1}^{1}|u|^{m+1} d x\right)\right]<\infty \text { and } \mathbb{E}\left[\exp \left(\epsilon[u]_{\alpha}^{2}\right)\right]<\infty .
$$


The following proposition, the proof of which is given in Appendix C, shows how to interpolate these two estimates to get optimal stochastic integrability for the supremum norm $\|u\|$.

Proposition 7.1. If $u \in C^{\alpha}(-1,1)$ and $u^{m+1}$ is integrable, then $u$ is bounded and we have the following interpolation:

$$
\left(\frac{\|u\|_{(-1,1)}}{2}\right)^{1+\alpha(m+1)} \leqslant \max \left\{[u]_{\alpha}\|u\|_{m+1}^{\alpha(m+1)},\|u\|_{m+1}^{1+\alpha(m+1)}\right\},
$$

where $\|\cdot\|_{m+1}$ refers to the $L^{m+1}$ norm on $[-1,1]$.

Since $2 \alpha<1$,

$$
\|u\|_{m+1}^{(m+1) \alpha}[u]_{\alpha} \leqslant\|u\|_{m+1}^{m+1}+[u]_{\alpha}^{2} .
$$

Hence, (7.2) implies that for $\epsilon$ small,

$$
\mathbb{E}\left[\exp \left(\epsilon\|u\|^{1+(m+1) \alpha}\right)\right]<\infty .
$$

On the other hand, from Theorem 3.1 and from Corollary 6.3, we get

$$
\mathbb{E}\left[\exp \left(\epsilon\left(2^{-\frac{2}{m-1}}\|u\|_{P_{\frac{1}{2}}}\right)^{2+(m-1) \alpha}\right)\right]<\infty .
$$

Therefore, for $\alpha \rightarrow \frac{1}{2}$, the exponents in (7.4) and (7.5) both converge to $\frac{m+3}{2}$.

\section{A Technical lemma}

For a multi-index $m \in \mathbb{N}^{d+1}$, denote $|m|=2 m_{0}+\sum_{k=1}^{d} m_{k}$ the parabolic index, and $m !=\prod_{k=0}^{d} m_{k} !$

Lemma A.1. Let $\Upsilon$ and $\Psi$ be two $C^{\infty}$ kernels supported in $B\left(0, R_{\Upsilon}\right)$ and $B\left(0, R_{\Psi}\right)$ respectively. Assume that for some odd integer $\beta>0$ and for all multi-indices $n$ with $|n| \leqslant \beta$ it holds $\int_{\mathbb{R}^{d+1}} z^{n} \Psi(z)=\delta_{n=0}$. For $R>0$ set $\bar{R}:=R+R_{\Upsilon}+2 R_{\Psi}$. Then for $\theta<\theta_{0}(\Psi)$ and for any function or distribution $\zeta$

$$
\|\zeta * \Upsilon\|_{B(0, R)} \lesssim \sup _{|n|=\beta+1, \beta+2} \int\left|\partial^{n} \Upsilon\right| \sup _{L=\theta^{k} \leqslant 1} L^{\beta}\left\|\zeta * \Psi_{L}\right\|_{B(0, \bar{R})}
$$

Here and in the proof $\lesssim$ means $\leqslant C(\Psi, \beta)$.

Proof. Define inductively $\omega^{0}=\Upsilon$ and $\omega^{k+1}=\omega^{k}-\Psi_{\theta^{k}} * \omega^{k}$. Since $\Psi$ cancels all polynomials of degree less than $\beta$, one can see from Taylor's formula that $\int \mid \omega^{k}-$ $\Psi_{\theta^{k}} * \omega^{k}\left|\lesssim \theta^{(\beta+1) k} \sup _{|n|=\beta+1, \beta+2} \int\right| \partial^{n} \omega^{k} \mid$ and $\int\left|\partial^{n}\left(\omega^{k}-\Psi_{\theta^{k}} * \omega^{k}\right)\right| \lesssim \int\left|\partial^{n} \omega^{k}\right|$, hence by induction

$$
\int\left|\omega^{k}\right| \lesssim C^{k} \theta^{(\beta+1) k} \sup _{|n|=\beta+1, \beta+2} \int\left|\partial^{n} \Upsilon\right| .
$$

This bound shows the convergence of the telescopic sum

$$
\Upsilon=\sum_{k=0}^{\infty} \Psi_{\theta^{k}} * \omega^{k}
$$

for $\theta$ small enough. 
We can then write

$$
\begin{aligned}
\|\zeta * \Upsilon\|_{B(0, R)} & =\left\|\sum_{k=0}^{\infty} \Psi_{\theta^{k}} * \omega^{k} * \zeta\right\|_{B(0, R)} \\
& \leqslant \sum_{k=0}^{\infty}\left\|\zeta * \Psi_{\theta^{k}}\right\|_{B(0, \bar{R})} \int\left|\omega^{k}\right| \\
& \leqslant \sup _{L=\theta^{k} \leqslant 1} L^{\beta}\left\|\zeta * \Psi_{L}\right\|_{B(0, \bar{R})} \sum_{k=0}^{\infty} C^{k} \theta^{k} \sup _{|n|=\beta+1, \beta+2} \int\left|\partial^{n} \Upsilon\right| .
\end{aligned}
$$

The following Lemma shows that the assumption of vanishing moments for $\Psi$ can be removed from the previous Lemma at the expense of making the domain on the right hand side yet a bit larger.

Lemma A.2. Let $\Upsilon$ and $\Psi$ be two $C^{\infty}$ kernels supported in $B\left(0, R_{\Upsilon}\right)$ and $B\left(0, R_{\Psi}\right)$ respectively. Let $\beta>0$ be an odd integer and for $R>0$ set $\bar{R}:=R+R_{\Upsilon}+5 R_{\Psi}$. Then for $\theta<\theta_{0}(\Psi)$ and for any function or distribution $\zeta$

$$
\|\zeta * \Upsilon\|_{B(0, R)} \lesssim \sup _{|n|=\beta+1, \beta+2} \int\left|\partial^{n} \Upsilon\right| \sup _{L=\theta^{k} \leqslant 1} L^{\beta}\left\|\zeta * \Psi_{L}\right\|_{B(0, \bar{R})} .
$$

Here and in the proof $\lesssim$ means $\leqslant C(\Psi, \beta)$.

Proof. For any $\beta>0$, we build from $\Psi$ a kernel $\Psi^{\prime}$ that satisfies the hypothesis of Lemma A.1.

We define $A_{n, m}=\int z^{n} \partial^{m} \Psi(z) d z$ and observe that since $\Psi$ is compactly supported and since $\int \Psi=1$, we have

$$
A_{n, m}=\left\{\begin{array}{cc}
0 & \text { if }|n| \leqslant|m|, n \neq m, \\
(-1)^{|m|} m ! & \text { if } n=m .
\end{array}\right.
$$

Hence for any $\beta>0,\left(A_{n, m}\right)_{|n|,|m| \leqslant \beta}$ is an invertible linear system. By continuity of the coefficients, for $r$ small enough,

$$
A_{n, m}^{r}=\int z^{n} \partial^{m}\left(\Psi_{r} * \Psi\right)(z) d z
$$

is also an invertible linear system. Hence, there exists coefficients $\left(a_{m}\right)_{|m| \leqslant \beta}$ such that

$$
\sum_{|m| \leqslant \beta} a_{m} \int z^{n} \partial^{m}\left(\Psi_{r} * \Psi\right)(z) d z=\left\{\begin{array}{cc}
1 & \text { if } n=0 \\
0 & \text { else. }
\end{array}\right.
$$

Set $\omega^{(0)}=\sum_{|m| \leqslant \beta} a_{m} \partial^{m} \Psi_{r}$ and $\Psi^{\prime}=\omega^{(0)} * \Psi$, then

$$
\int z^{n} \Psi^{\prime}(z) d z=\left\{\begin{array}{cc}
1 & \text { if } n=0 \\
0 & \text { for } 0<|n|<\beta .
\end{array}\right.
$$

We can therefore apply Lemma A.1 with $\Psi^{\prime}$ to get

$$
\|\zeta * \Upsilon\|_{B(0, R)} \lesssim \sup _{|n|=\beta+1, \beta+2} \int\left|\partial^{n} \Upsilon\right| \sup _{L=\theta^{k} \leqslant 1} L^{\beta}\left\|\zeta * \Psi_{L}\right\|_{B\left(0, R+R \Upsilon+2 R_{\Psi^{\prime}}\right)} .
$$

We have also

$$
\begin{aligned}
\left\|\zeta * \Psi_{L}^{\prime}\right\|_{B\left(0, R+R \Upsilon+2 R_{\Psi^{\prime}}\right)} & =\left\|\zeta * \Psi_{L} * \omega_{L}^{(0)}\right\|_{B\left(0, R+R_{\Upsilon}+2 R_{\Psi^{\prime}}\right)} \\
& \leqslant\left\|\zeta * \Psi_{L}\right\|_{B\left(0, R+R_{\Upsilon}+5 R_{\Psi}\right)} \int\left|\omega^{(0)}\right| .
\end{aligned}
$$




\section{B Low regularity Schauder estimate}

We give here a proof of a low regularity Schauder estimate in our setting.

Lemma B.1. Let $u$ be a function in $C^{\alpha}$ for a $\alpha \in(0,1)$ and let $f:=\left(\partial_{t}-\Delta\right) u$. There exists a constant $C=C(\alpha, d)$ such that

$$
[u]_{\alpha} \leqslant C \sup _{0<L<\infty} L^{2-\alpha}\left\|f_{L}\right\| .
$$

Proof. Throughout the proof $\lesssim$ will denote a bound up to a multiplicative constant, which may change from line to line, but which always depends only on $\alpha$ and $d$. Define $N=\sup _{L \leqslant 1} L^{2-\alpha}\left\|f_{L}\right\|$. Since $(\cdot)_{L}$ denotes the convolution with a smooth kernel, it commutes with derivatives. We know that for $L<1$, for any $l \in \operatorname{span}\left\{1, x_{i}, i \in\{1, \ldots, d\}\right\}$, we have on $\mathbb{R} \times \mathbb{R}^{d}$,

$$
\left(\partial_{t}-\Delta\right)\left(u_{L}-l\right)=f_{L}
$$

For $z_{0} \in B(0,1)$, for some $S>0$ to be fixed below, define $v_{>}$as the solution to

$$
\left(\partial_{t}-\Delta\right) v_{>}=\mathbf{1}_{\left\{B\left(z_{0}, S\right)\right\}} f_{L},\left.\quad v_{>}\right|_{\partial B\left(z_{0}, S\right)}=0,
$$

where $\partial B\left(z_{0}, S\right)=\left\{z=(t, x), d\left(z, z_{0}\right)=S, t \leqslant t_{0}\right\}$ is the parabolic boundary of $B\left(z_{0}, S\right)$. The first interesting inequality we get from standard heat equation estimates [13, Cor.8.1.5] is

$$
\left\|v_{>}\right\| \lesssim S^{2}\left\|f_{L}\right\| \leqslant S^{2} L^{\alpha-2} N .
$$

Define $v_{<}=u_{L}-v_{>}$. As $\left(\partial_{t}-\Delta\right) v_{<}=0$ on $B\left(z_{0}, S\right)$ for any differential operator $D \in\left\{\partial_{t}, \partial_{i} \partial_{j}, i, j \in\{1, \ldots, d\}\right\}$,

$$
\left\|D v_{<}\right\|_{B_{\frac{S}{2}}} \lesssim S^{-2} \inf _{l}\left\|u_{L}-l\right\|_{B\left(z_{0}, S\right)},
$$

where $l$ runs over all function spanned by 1 and $x_{i}, i \in\{1, \ldots, d\}$. Therefore, for any $R<\frac{S}{2}$, for the same range of operator $D$, for a suitably chosen $l_{R} \in \operatorname{span}\left\{1, x_{i}, i \in\{1, \ldots, d\}\right\}$,

$$
\left\|v_{<}-l_{R}\right\|_{B\left(z_{0}, R\right)} \leqslant R^{2}\left\|D v_{<}\right\|_{B\left(z_{0}, R\right)} \lesssim\left(\frac{R}{S}\right)^{2} \inf _{l}\left\|u_{L}-l\right\|_{B\left(z_{0}, S\right)} .
$$

Using the definition of $v_{<}$and the triangle inequality,

$$
\left\|u_{L}-l_{R}\right\|_{B\left(z_{0}, R\right)}-\left\|v_{>}\right\|_{B\left(z_{0}, R\right)} \lesssim\left(\frac{R}{S}\right)^{2} \inf _{l}\left\|u_{L}-l\right\|_{B\left(z_{0}, S\right)} .
$$

From (B.2),

$$
\begin{aligned}
\frac{1}{R^{\alpha}}\left\|u_{L}-l_{R}\right\| \lesssim & \left(\frac{R}{S}\right)^{2-\alpha} \frac{1}{S^{\alpha}} \inf _{l}\left\|u_{L}-l\right\|_{B\left(z_{0}, S\right)} \\
& +\left(\frac{S}{L}\right)^{2}\left(\frac{L}{R}\right)^{\alpha} N .
\end{aligned}
$$

Furthermore, from (3.7) we get,

$$
\frac{1}{R^{\alpha}}\left\|u-l_{R}\right\|_{B\left(z_{0}, R\right)} \lesssim \frac{1}{R^{\alpha}}\left\|u_{L}-l_{R}\right\|_{B\left(z_{0}, R\right)}+\left(\frac{L}{R}\right)^{\alpha}[u]_{\alpha} .
$$

Similarly, for any $l \in \operatorname{span}\left\{1, x_{i}, i \in\{1, \ldots, d\}\right\}$

$$
\frac{1}{S^{\alpha}}\left\|u_{L}-l\right\|_{B\left(z_{0}, S\right)} \lesssim \frac{1}{S^{\alpha}}\|u-l\|_{B\left(z_{0}, S\right)}+\left(\frac{L}{S}\right)^{\alpha}[u]_{\alpha} .
$$


Hence for $0<\epsilon<1$, for $L=\epsilon R=\epsilon^{2} S$, (B.3) and (B.4), (B.5) give:

$$
\begin{aligned}
\frac{1}{R^{\alpha}} \inf _{l}\|u-l\|_{B\left(z_{0}, R\right)} \lesssim & \epsilon^{2-\alpha} \frac{1}{S^{\alpha}} \inf _{l}\|u-l\|_{B\left(z_{0}, S\right)} \\
& +\left(\epsilon^{\alpha}+\epsilon^{2 \alpha}\right)[u]_{\alpha}+\epsilon^{\alpha-4} N .
\end{aligned}
$$

Note that $[u]_{\alpha} \sim \sup _{z_{0}} \sup _{S} \frac{1}{S^{\alpha}} \inf _{l}\|u-l\|_{B\left(z_{0}, S\right)}$, hence

$$
[u]_{\alpha} \lesssim\left(\epsilon^{2-\alpha}+\epsilon^{\alpha}+\epsilon^{2 \alpha}\right)[u]_{\alpha}+\epsilon^{\alpha-4} N .
$$

By making $\epsilon$ small enough, we can absorb $[u]_{\alpha}$ in the right hand side of (B.8) into the left hand side, concluding the proof of the Schauder estimate (B.1).

Corollary B.2. Let $f$ be compactly supported in $B(0, R)$ and let $u$ be the unique bounded solution to $\left(\partial_{t}-\Delta\right) u=f$ which vanishes for $t \leqslant-R^{2}$. Then for $\alpha \in(0,1)$ there exists a constant $C=C(\alpha, d)$ such that

$$
[u]_{\alpha} \leqslant C \sup _{0<L \leqslant 2 R} L^{2-\alpha}\left\|f_{L}\right\|,
$$

and

$$
\|u\| \leqslant C R^{\alpha} \sup _{L \leqslant 2 R} L^{2-\alpha}\left\|f_{L}\right\| .
$$

Proof. By a scaling argument, we can show the result fo $R=\frac{1}{2}$. We first show an equivalence of kernels by applying Lemma A.2 with $\beta>1-\alpha$ and $\Upsilon=\Psi_{L}-\Psi_{*} \Psi_{L-1}$. Since $f$ has compact support, we do not need to keep track of the domains. There exists a $\theta<1$ and a constant $C=C(\Psi, \beta)$ such that:

$$
\left\|f_{L}-\left(f_{1}\right)_{L-1}\right\| \leqslant C \sup _{|n|=\beta+1, \beta+2} \int\left|\partial^{n}\left(\Psi_{L}-\Psi * \Psi_{L-1}\right)\right| \sup _{S=\theta^{k}} S^{\beta}\left\|f_{S}\right\|
$$

Therefore, we can write

$$
\begin{array}{r}
L^{2-\alpha}\|f\|_{L} \leqslant L^{2-\alpha}\left\|\left(f_{1}\right)_{L-1}\right\|+L^{2-\alpha} C L^{-\beta-1} \sup _{S=\theta^{k}} S^{\beta}\left\|f_{S}\right\| \\
\leqslant L^{2-\alpha}(L-1)^{-d-2}\left\|f_{1}\right\|+C L^{1-\alpha-\beta} \sup _{S \leqslant 1} S^{2-\alpha}\left\|f_{S}\right\| \\
\leqslant\left(L^{2-\alpha}(L-1)^{-d-2}+C L^{1-\alpha-\beta}\right) \sup _{S \leqslant 1} S^{2-\alpha}\left\|f_{S}\right\| .
\end{array}
$$

Therefore the supremum can be taken over scales $L \leqslant 2 R$. In the proof of Lemma B.1, the only place where the hypothesis $[u]_{\alpha}<\infty$ was used was in (B.8). This assumption can be removed as by regularising the equation first, we have that uniformly for any $\tau>0$,

$$
\left[u_{\tau}\right]_{\alpha} \lesssim \sup _{L \leqslant 2 R} L^{2-\alpha}\left\|\left(f_{\tau}\right)_{L}\right\| \leqslant \sup _{L \leqslant 2 R} L^{2-\alpha}\left\|f_{L}\right\|,
$$

and as $u$ is continuous, we can pass to the limit for $\tau \rightarrow 0$.

For (B.10), we write $u=u_{R}+\left(u-u_{R}\right)$. For the second term we get

$$
\left\|u-u_{R}\right\| \leqslant R^{\alpha}[u]_{\alpha} \stackrel{\text { (B.9) }}{\lesssim} R^{\alpha} \sup _{0<L \leqslant 2 R} L^{2-\alpha}\left\|f_{L}\right\| .
$$

For the smooth part $u_{R}$ we write $\left(\partial_{t}-\Delta\right) u_{R}=f_{R}$ we use a standard $L^{\infty}$ estimate for the heat equation with compactly supported right hand side [13, Thm 8.4.2] and get

$$
\left\|u_{R}\right\| \lesssim R^{2}\left\|f_{R}\right\| \lesssim R^{\alpha} \sup _{0<L \leqslant 2 R} L^{2-\alpha}\left\|f_{L}\right\| .
$$




\section{Proof of Proposition 7.1}

For any interval $I \subset[-1,1]$,

$$
|u(t)|-\frac{1}{|I|}\left|\int_{I} u(s) d s\right| \leqslant \frac{1}{|I|} \int_{I}|u(t)-u(s)| d s \leqslant \frac{1}{|I|} \int_{I}[u]_{\alpha, I}|t-s|^{\alpha} .
$$

If $t \in I,|t-s|^{\alpha} \leqslant I^{\alpha}$. We can apply Jensen's inequality.

$$
|u(t)| \leqslant\left(\frac{1}{|I|} \int_{I}|u(s)|^{m+1} d s\right)^{\frac{1}{m+1}}+|I|^{\alpha}[u]_{\alpha, I} .
$$

And since this is true for any $I \subset[-1,1]$, we have for any choice of $0<x \leqslant 2$,

$$
\|u\|_{J} \leqslant x^{-\frac{1}{m+1}}\|u\|_{m+1}+x^{\alpha}[u]_{\alpha, J} .
$$

If $\|u\|_{m+1} \geqslant[u]_{\alpha}$ then choose $x=1$ to get $\|u\|_{(-1,1)} \leqslant 2\|u\|_{m+1}$. Else choose $x=$ $\left(\|u\|_{m+1} /[u]_{\alpha}\right)^{\frac{m+1}{1+\alpha(m+1)}} \leqslant 1$ and get $\|u\|_{(-1,1)} \leqslant 2[u]_{\alpha}^{\frac{1}{1+\alpha(m+1)}}\|u\|_{m+1}^{\frac{\alpha(m+1)}{1+\alpha(m+1)}}$. In conclusion,

$$
\|u\|_{(-1,1)} \leqslant 2 \max \left\{\|u\|_{m+1},[u]_{\alpha}^{\frac{1}{1+\alpha(m+1)}}\|u\|_{m+1}^{\frac{\alpha(m+1)}{1+\alpha(m+1)}}\right\} .
$$

\section{References}

[1] S. Albeverio, H. Kawabi, and M. Röckner. Strong uniqueness for both dirichlet operators and stochastic dynamics to gibbs measures on a path space with exponential interactions. Journal of Functional Analysis, 262(2):602-638, 2012. MR-2854715

[2] S. Albeverio and S. Kusuoka. The invariant measure and the flow associated to the $\phi_{3}^{4}$-quantum field model. arXiv preprint arXiv:1711.07108, 2017.

[3] H. Bahouri, J.-Y. Chemin, and R. Danchin. Fourier analysis and nonlinear partial differential equations, volume 343. Springer Science \& Business Media, 2011. MR-2768550

[4] S. Cerrai. Second order PDE's in finite and infinite dimension: a probabilistic approach, volume 1762. Springer Science \& Business Media, 2001. MR-1840644

[5] S. Cerrai. Stochastic reaction-diffusion systems with multiplicative noise and non-Lipschitz reaction term. Probability Theory and Related Fields, 125(2):271-304, 2003. MR-1961346

[6] G. Da Prato and A. Debussche. Strong solutions to the stochastic quantization equations. The Annals of Probability, 31(4):1900-1916, 2003. MR-2016604

[7] G. Da Prato and J. Zabczyk. Ergodicity for infinite dimensional systems, volume 229. Cambridge University Press, 1996. MR-1417491

[8] G. Da Prato and J. Zabczyk. Stochastic equations in infinite dimensions. Cambridge University Press, 2014. MR-3236753

[9] M. Gubinelli and M. Hofmanová. Global solutions to elliptic and parabolic $\phi^{4}$ models in euclidean space. arXiv preprint arXiv:1804.11253, 2018. MR-3951704

[10] M. Hairer. An introduction to stochastic pdes. arXiv preprint arXiv:0907.4178, 2009. MR2832168

[11] M. Hairer. A theory of regularity structures. Inventiones Mathematicae, 198(2):269-504, 2014. MR-3274562

[12] K. Iwata. An infinite dimensional stochastic differential equation with state space $C(\mathbb{R})$. Probability Theory and Related Fields, 74(1):141-159, 1987. MR-0863723

[13] N. V. Krylov. Lectures on elliptic and parabolic equations in Hölder spaces. Number 12. American Mathematical Soc., 1996. MR-1406091

[14] A. Moinat and H. Weber. Space-time localisation for the dynamic $\phi_{3}^{4}$ model. Communications on Pure and Applied Mathematics, to appear 2019+.

[15] J.-C. Mourrat and H. Weber. The dynamic $\phi_{3}^{4}$ model comes down from infinity. Communications in Mathematical Physics, 356(3):673-753, 2017. MR-3719541 
[16] J.-C. Mourrat, H. Weber, et al. Global well-posedness of the dynamic $\phi^{4}$ model in the plane. The Annals of Probability, 45(4):2398-2476, 2017. MR-3693966

[17] W. F. Osgood. Beweis der Existenz einer Lösung der Differentialgleichung $\frac{d y}{d x}=f(x, y)$ ohne Hinzunahme der Cauchy-Lipschitz'schen Bedingung. Monatshefte für Mathematik und Physik, 9(1):331-345, 1898. MR-1546565

[18] F. Otto and H. Weber. Quasilinear spdes via rough paths. Archive for Rational Mechanics and Analysis, 232(2):873-950, 2019. MR-3925533

[19] J. Smoller. Shock waves and reaction - diffusion equations, volume 258. Springer Science \& Business Media, 2012. MR-1301779

[20] P. Tsatsoulis and H. Weber. Spectral gap for the stochastic quantization equation on the 2-dimensional torus. Ann. Inst. H. Poincaré Probab. Statist., 54(3):1204-1249, 082018. MR-3825880

[21] J. B. Walsh. An introduction to stochastic partial differential equations. In École d'Été de Probabilités de Saint Flour XIV-1984, pages 265-439. Springer, 1986. MR-0876085 